\title{
Methylene blue stimulates substrate-level phosphorylation catalysed by succinyl-CoA ligase in the citric acid cycle
}

\author{
T. Komlódi, L. Tretter* \\ Department of Medical Biochemistry, MTA-SE Laboratory for Neurobiochemistry, Semmelweis University, 37-47 Tuzolto St., Budapest, 1094, Hungary
}

\section{A R T I C L E IN F O}

Article history:

Received 30 January 2017

Received in revised form 5 May 2017

Accepted 7 May 2017

Available online xxx

\section{Keywords:}

Methylene blue

Neuroprotection

Mitochondria

Citric acid cycle

Substrate-level phosphorylation

Neurodegeneration

Compounds:

Methylene blue (PubChem CID: 6099)

$\alpha$-ketoglutarate (PubChem CID: 51)

Succinate (PubChem CID: 1110)

Malate (PubChem CID: 222656)

Oligomycin (PubChem CID: 78358496)

$\mathrm{P}^{1}, \mathrm{P}^{5}$-di(adenosine-5 $\left.5^{\prime}\right)$ pentaphosphate (Pub-

Chem CID: 6419779)

Carboxyatractilozide (PubChem CID:

101834999)

Carbonylcyanide-p-triflouromethoxy-phenylhydrazone (PubChem CID: 3330)

\section{A B S T R A C T T I T L E}

Methylene blue (MB), a potential neuroprotective agent, is efficient in various neurodegenerative disease models. Beneficial effects of MB have been attributed to improvements in mitochondrial functions. Substrate-level phosphorylation (SLP) results in the production of ATP independent from the ATP synthase (ATP-ase). In energetically compromised mitochondria, ATP produced by SLP can prevent the reversal of the adenine nucleotide translocase and thus the hydrolysis of glycolytic ATP. The aim of the present study was to investigate the effect of MB on mitochondrial SLP catalysed by succinyl-CoA ligase. Measurements were carried out on isolated guinea pig cortical mitochondria respiring on $\alpha$-ketoglutarate, glutamate, malate or succinate. The mitochondrial functions and parameters like ATP synthesis, oxygen consumption, membrane potential, and $\mathrm{NAD}(\mathrm{P}) \mathrm{H}$ level were followed online, in parallel with the redox state of MB. SLP-mediated ATP synthesis was measured in the presence of inhibitors for ATP-ase and adenylate kinase. In the presence of the ATP-ase inhibitor oligomycin MB stimulated respiration with all of the respiratory substrates. However, the rate of ATP synthesis increased only with substrates $\alpha$-ketoglutarate and glutamate (forming succinyl-CoA). MB efficiently stimulated SLP and restored the membrane potential in mitochondria also with the combined inhibition of Complex I and ATP synthase. ATP formed by SLP alleviated the energetic insufficiency generated by the lack of oxidative phosphorylation. Thus, the MB-mediated stimulation of SLP might be important in maintaining the energetic competence of mitochondria and in preventing the mitochondrial hydrolysis of glycolytic ATP. The mitochondrial effects of MB are explained by the ability to accept electrons from reducing equivalents and transfer them to cytochrome $\mathrm{c}$ bypassing the respiratory Complexes I and III.

(C) 2016 Published by Elsevier Ltd.

\section{Introduction}

Methylene blue (MB) (for structure, see: Supplemental Data, Fig. 1) was identified as an antimalarial drug in 1891 (Guttman and Ehrlich, 1891). In the last century, MB has been used for the treatment of methemoglobinaemia (Mansouri and Lurie, 1993), carbon monoxide and cyanide poisoning (Draize, 1933). It displayed benefi-

\footnotetext{
Abbreviations: AK, adenylate kinase; ANT, adenine nucleotide translocase; $\alpha-\mathrm{KG}$, alpha-ketoglutarate; $\alpha-\mathrm{KGDHc}$, alpha-ketoglutarate dehydrogenase complex; AP5, $\mathrm{P}^{1}, \mathrm{P}^{5}$-di(adenosine-5') pentaphosphate; BSA, bovine serum albumin; CAT, carboxyatractilozide; $\Delta \psi_{\mathrm{m}}$, mitochondrial membrane potential; FCCP, carbonylcyanide- $p$-triflouromethoxy-phenylhydrazone; $\mathrm{MDH}$, malate dehydrogenase; MB, methylene blue; OXPHOS, oxidative phosphorylation; SLP, substrate-level phosphorylation; TCA cycle, tricarboxylic acid cycle

* Corresponding author.

Email address: tretter.laszlo@med.semmelweis-univ.hu (L. Tretter)
}

cial effects in neurodegenerative disease models (Eckert et al., 2012; Paban et al., 2014; Sontag et al., 2012; Zhang et al., 2006) and stroke (Di et al., 2015). In Alzheimer's disease models MB augmented the activity of Complex IV and stimulated the synthesis of heme (Atamna et al., 2008).

The U.S. Food and Drug Administration (FDA) approved MB for the emergency treatment of methemoglobinaemia (FDA Reference ID: 3914270). MB provides an alternative mechanism for electron transport by transferring electrons from NADH (Wen et al., 2011) and $\mathrm{FADH}_{2}$ (Dixon, 1971) to cytochrome c (Atamna et al., 2008; McCord and Fridovich, 1970) or molecular oxygen (Tretter et al., 2014). The aromatic ring of $\mathrm{MB}$ provides high lipophilicity and consequently good membrane permeability while the positive charge on MB secures mitochondrial membrane potential $\left(\Delta \psi_{\mathrm{m}}\right)$-dependent accumulation in the mitochondrial matrix (Gabrielli et al., 2004). As we have previously reported (Tretter et al., 2014), MB stimulates ATP synthesis, rescues $\Delta \psi_{\mathrm{m}}$ and increases $\mathrm{Ca}^{2+}$ uptake in mitochondria with impaired Complexes I and III. 
In mitochondria, ATP can be produced via three mechanisms: i) oxidative phosphorylation (OXPHOS), ii) SLP in the tricarboxylic acid (TCA) cycle (catalyzed by succinyl-CoA ligase), and iii) the adenylate kinase (AK) reaction (2 ADP $\leftrightarrow$ ATP + AMP) (Bruns and Regina, 1977; Nobumoto et al., 1998; Panayiotou et al., 2014). Mitochondrial SLP is attributed to succinyl-CoA ligase, an enzyme of the TCA cycle having two isoforms: an ATP-forming isoform (succinyl-CoA $+\mathrm{ADP}+\mathrm{P}_{\mathrm{i}} \leftrightarrow$ succinate $+\mathrm{CoA}-\mathrm{SH}+\mathrm{ATP}(\mathrm{EC}: 6.2 .1 .5)$ ) and a GTP-forming isoform (succinyl-CoA + GDP $+\mathrm{P}_{\mathrm{i}} \leftrightarrow$ succinate + CoA-SH + GTP (EC: 6.2.1.4)); both reactions are reversible (Johnson et al., 1998). In rodent brain the ATP-forming isoform is dominating (Lambeth et al., 2004; Ostergaard, 2008). SLP is considered to be an alternative mechanism for ATP synthesis inside the mitochondrion (Heldt and Schwalbach, 1967); it is practically independent of the respiratory chain and the mitochondrial proton motive force. Under physiological conditions SLP has an essential role in ATP production during thermogenesis in brown-adipose tissue (Rafael et al., 1974; Smith et al., 1966). Furthermore, SLP has a significant role when OXPHOS is impaired (Chinopoulos, 2011b). Recently a new and important role has also been attributed to matrix SLP. Mitochondria have been known to both synthesize and hydrolyze ATP (Nicholls and Ferguson, 2013). The ATP hydrolytic activity of mitochondria is typically associated with the hydrolysis of glycolytic ATP and the complete depletion of cellular ATP. In order to hydrolyze glycolytic ATP both the ATP synthase and adenine nucleotide translocase (ANT) have to work in their reverse modes. However, the reversals of ATP synthase and ANT have been shown to occur nonsimultaneously because the reversal potentials of the two functions are dissimilar (Chinopoulos, 2011b). ATP production via matrix SLP may prevent the reversal of ANT and thus could inhibit the mitochondrial utilisation of cytosolic ATP (Chinopoulos, 2011a). Metabolic intermediates inside the mitochondrion can be classified as promoters and non-promoters of the SLP (Kiss et al., 2013). To selectively measure SLP activity both OXPHOS and AK-catalyzed ATP production have to be minimized.

The aim of this study was to examine the effects of MB on matrix SLP and mitochondrial bioenergetics in the presence of the ATP synthase inhibitor oligomycin and the adenylate kinase inhibitor AP5 (see structure: Supplemental Data, Fig. 2) in isolated, intact brain mitochondria. The concentrations of $\mathrm{MB}$ applied in this study $(300 \mathrm{nM}-2 \mu \mathrm{M})$ corresponded to the blood levels of MB detected after i.v. administration (Peter et al., 2000; Schirmer et al., 2011). Isolated guinea pig brain mitochondria were supported with various respiratory substrates including $\alpha$-ketoglutarate $(\alpha-K G)$, glutamate, malate, or succinate. MB enhanced the rate of mitochondrial SLP in the presence of glutamate or $\alpha-K G$, but did not stimulate the SLP with succinate or malate. In the presence of the respiratory Complex I inhibitor rotenone MB was still able to stimulate the SLP and could restore the dissipated $\Delta \psi_{\mathrm{m}}$. The potentially neuroprotective drug MB could have beneficial effects in OXPHOS-inhibited mitochondria.

\section{Materials and methods}

\subsection{Chemicals}

All laboratory chemicals except for ADP were purchased from Sigma (St. Louis, MO, USA). ADP was purchased from Merck (Darmstadt, Germany).

\subsection{Preparation of brain mitochondria}

Mitochondria were isolated from the brain cortex of albino male guinea pigs using a Percoll gradient as previously described (Rosenthal et al., 1987; Tretter and Adam-Vizi, 2007). Animal experiments were performed in accordance with the guidance of the National Institute of Health (USA) for the care and use of laboratory animals. Brain was homogenised in ice cold buffer "A" (in mM: 225 mannitol, 75 sucrose, 5 HEPES, 1 EGTA, $\mathrm{pH}$ 7.4) and centrifuged for 3 min at $1300 \mathrm{~g}$. Subsequently, the supernatant was centrifuged for $10 \mathrm{~min}$ at $20,000 \mathrm{~g}$ and the resultant pellet was resuspended in $15 \%$ Percoll and layered on a discontinuous gradient (40 and 23\%) of Percoll. This gradient was then centrifuged for $8 \mathrm{~min}$ at $30,700 \mathrm{~g}$ using no brake at the end. After resuspending the lower fraction in buffer "A", the solution was centrifuged at $16,600 \mathrm{~g}$ for $10 \mathrm{~min}$, the resultant pellet was resuspended in buffer "A" and subsequently centrifuged again at $6300 \mathrm{~g}$ for $10 \mathrm{~min}$. After the supernatant was discharged, the pellet was resuspended in buffer "B", which contained no EGTA (in mM: 225 mannitol, 75 sucrose, 5 HEPES, pH 7.4). Protein concentration was determined by the modified biuret method (Bradford, 1976). Unless otherwise indicated, $0.1 \mathrm{mg} / \mathrm{ml}$ mitochondrial protein concentration was used throughout the experiments.

\subsection{Measurement of mitochondrial respiration}

Oxygen consumption was measured by high-resolution respirometry using an Oxygraph-2k instrument (OROBOROS Instruments, Innsbruck, Austria). Standardised two-point calibrations of the polarographic oxygen sensor were applied (Pesta and Gnaiger, 2012). Measurements were performed in an assay medium containing (in $\mathrm{mM}$ ): 0.1 EGTA, $125 \mathrm{KCl}, 20 \mathrm{HEPES}, 2 \mathrm{~K}_{2} \mathrm{HPO}_{4}, 1 \mathrm{MgCl}_{2}$, and $0.025 \%$ BSA (fatty acid-free) at $\mathrm{pH} 7.0$. In all experiments, $200 \mu \mathrm{M}$ AP5 was used to inhibit AK isoforms. Data were digitally recorded using the DatLab4 software (OROBOROS Instruments, Innsbruck, Austria).

\subsection{Measurement of ATP production}

To measure ATP production, a coupled enzyme assay applying hexokinase and glucose-6-phosphate dehydrogenase was used (Williamson and Corkey, 1979). The assay medium described above was supplemented with $3 \mathrm{mM} \mathrm{NADP}^{+}, 1.5 \mathrm{U}$ hexokinase, $0.5 \mathrm{U}$ glucose-6-phosphate dehydrogenase, $5 \mathrm{mM}$ glucose, and $200 \mu \mathrm{M}$ AP5 $\left(\mathrm{P}^{1}, \mathrm{P}^{5}\right.$-Di(adenosine-5') pentaphosphate; an inhibitor of adenylate kinase) in $2 \mathrm{ml}$ total volume (Melnick et al., 1979). Throughout the measurements, $0.05 \mathrm{mg} / \mathrm{ml}$ mitochondria and $2 \mathrm{mM}$ ADP were used. In the presence of mitochondria and the applied respiratory substrate(s) ADP was phosphorylated to ATP. ATP left mitochondria via ANT. Hexokinase in the medium phosphorylated glucose to glucose-6-phosphate using ATP. Then, glucose-6-phosphate was oxidized to 6-phosphogluconate by glucose-6-phospate dehydrogenase with the concomitant reduction of $\mathrm{NADP}^{+}$to NADPH. NADPH formation was detected as proportional to ATP released from the mitochondria. Absorbance of NADPH $\left(\varepsilon=6220 \mathrm{M}^{-1} \mathrm{~cm}^{-1}\right)$ was recorded at $340 \mathrm{~nm}$, $37^{\circ} \mathrm{C}$ using a JASCO V-650 spectrophotometer (ABL\&E-JASCO, Tokyo, Japan). Measurements were calibrated using ATP standards. To detect mitochondrial SLP, the following protocol was used: ADP was first added to the mitochondria and subsequently, OXPHOS was initiated by adding a respiratory substrate. Then, ATP synthase was inhibited by oligomycin. Afterwards, MB was added. At the end of 
each experiment, ANT was blocked by the specific inhibitor carboxyatractylozide (CAT).

\subsection{Measurement of mitochondrial membrane potential $\left(\Delta \psi_{m}\right)$}

The $\Delta \psi_{\mathrm{m}}$ was detected using safranin $\mathrm{O}$, a positively charged dye, which undergoes an optical shift upon membrane potential distribution between the medium and the mitochondrial matrix (Akerman and Wikstrom, 1976; Figueira et al., 2012). Safranin ( $2 \mu \mathrm{M})$ fluorescence was measured with a Hitachi F-4500 spectrofluorimeter (Hitachi High Technologies, Maidenhead, UK) at 495 and $585 \mathrm{~nm}$ excitation and emission wavelengths at $37^{\circ} \mathrm{C}$, respectively. The assay medium was the same as that for mitochondrial respiration. $0.1 \mathrm{mg} / \mathrm{ml}$ mitochondrial protein concentration was used in the experiments.

\subsection{Measurement of $M B$ absorbance}

Oxidised MB's absorbance was detected at $660 \mathrm{~nm}$ using a JASCO $\mathrm{V}-650$ spectrophotometer. $0.1 \mathrm{mg} / \mathrm{ml}$ mitochondrial protein concentration was used throughout the experiments. Measurements were performed in an assay medium containing (in mM): 0.1 EGTA, $125 \mathrm{KCl}$, $20 \mathrm{HEPES}, 2 \mathrm{~K}_{2} \mathrm{HPO}_{4}, 1 \mathrm{MgCl}_{2}$ and $0.025 \% \mathrm{BSA}$ (fatty acid-free) at $\mathrm{pH}$ 7.0. $\mathrm{MB}(2 \mu \mathrm{M})$ was added at the beginning of each experiment.

\subsection{Statistics}

Data are presented as the means \pm S.E.M. Significant differences were evaluated with the one-way ANOVA procedure followed by the Holm-Sidak's post-hoc test for multiple comparisons, or for data not following normal distribution ANOVA on ranks Kruskar Wallis test was applied. Values of $p<0.05$ were considered to be significant.

\section{Results}

\subsection{The contribution of adenylate kinase activity to mitochondrial ATP production}

In preliminary experiments, the addition of ADP to isolated mitochondria in an ATP assay test resulted in a consistent, elongated $\mathrm{NAD}(\mathrm{P}) \mathrm{H} \mathrm{H}^{+}$production in the absence of respiratory substrates. This OXPHOS and SLP-independent ATP production could be attributed to the presence of AKs (Barkulis and Lehninger, 1951; Panayiotou et al., 2014). This high ATP synthetic activity could add a significant "background" to the ATP measurements. To eliminate this activity, an inhibitor of AKs, AP5 was used (Melnick et al., 1979). After titrating the AK-dependent ATP production with AP5, $200 \mu \mathrm{M}$ AP5 was the most effective concentration to inhibit mitochondrial AK activity without impairing mitochondrial respiration. In non-energised mitochondria addition of $200 \mu \mathrm{M}$ AP5 resulted a $83 \%$ decrease of the rate of ATP production during the first $3 \mathrm{~min}$ (Fig. 1.) The residual ATP production $(22-23 \mathrm{nmol} / \mathrm{min} / \mathrm{mg}$ protein) was also AP5 sensitive, but the higher AP5 concentrations were toxic for mitochondria (not shown). Carboxyatractilozide (CAT) an inhibitor of the ATP/ ADP exchange transporter (adenine nucleotide translocase; ANT) resulted in a small decrease in ATP production both in the presence or absence of AP5 indicating that most of the AK activity is localized on the outer surface of the inner mitochondrial membrane (Fig. 1). Off-target effects of AP5 were also detected, but those have not influenced the validity of the results. These off-target effects are described in the Supplementary material.

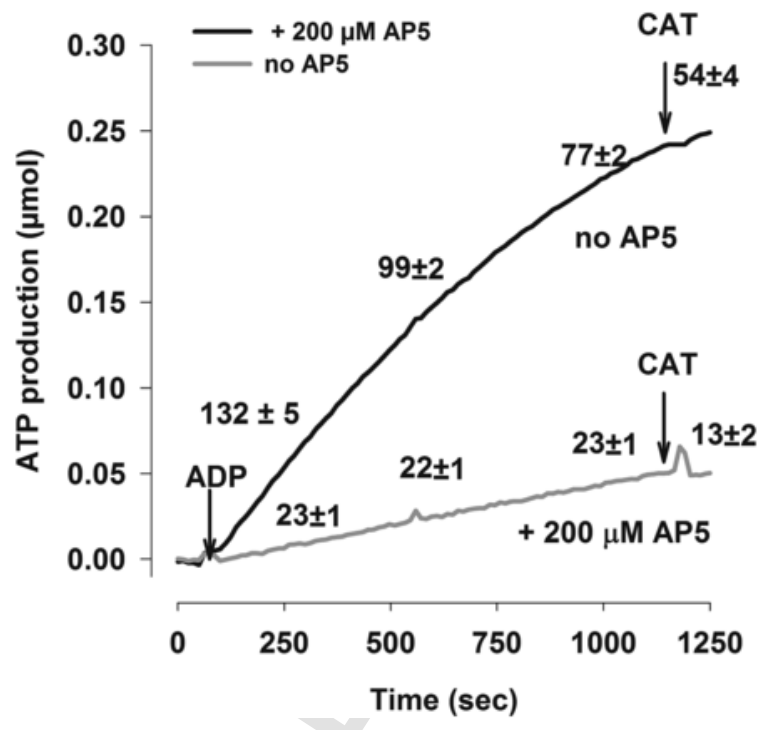

Fig. 1. The rate of ATP production as a function of time in the presence or absence of $200 \mu \mathrm{M}$ AP5 in mitochondria devoid of respiratory substrates. Mitochondria $(0.1 \mathrm{mg} /$ $\mathrm{ml})$ were incubated in the standard medium as described under Materials and methods. ADP $(2 \mathrm{mM})$ was given as indicated. Numbers measured as the rate of ATP synthesis are in $\mathrm{nmol} / \mathrm{min} / \mathrm{mg}$ protein mean $\pm \operatorname{SEM}(n>4)$. Trace a shows the rate of ATP synthesis in the absence of AP5; trace $b$ the rate of ATP synthesis in the presence of AP5.

\subsection{The effect of $M B$ on the rate of ATP synthesis (substrate level phosphorylation) in oxidative phosphorylation-inhibited mitochondria}

In a previous study by us (Tretter et al., 2014) in Complex I or complex III-compromised mitochondria MB, applied at 1 or $2 \mu \mathrm{M}$ concentrations significantly stimulated the OXPHOS-mediated ATP generation; in the present study, $300 \mathrm{nM}, 1 \mu \mathrm{M}$ or $2 \mu \mathrm{M}$ MB was applied. The contribution of SLP to gross ATP production was calculated by subtracting the ADP-evoked ATP production measured in the absence of the respiratory substrate from the ATP production detected in the presence of ADP, respiratory substrate and oligomycin. Oligomycin, an inhibitor of the mitochondrial ATP synthase (also known as $\mathrm{F}_{0} \mathrm{~F}_{1}$ ATP-ase) blocks OXPHOS, hence in its presence ATP synthesis can be attributed exclusively to the AK activity plus SLP.

In $\alpha-K G$-supported mitochondria, the basal SLP (SLP in the presence of oligomycin, but in the absence of $\mathrm{MB}$ ) was found to be $(47 \pm 3)-(23 \pm 1)=24 \pm 2 \mathrm{nmol} / \mathrm{min} / \mathrm{mg}$ protein (Fig. $2 \mathrm{~A}$ and B). MB stimulated the mitochondrial ATP synthesis with $\alpha-K G$ as respiratory substrate in a dose-dependent manner (Fig. 2B). The rate of ATP production in the presence of oligomycin, ADP and $\alpha-\mathrm{KG}$ was increased by $26 \%$ after $300 \mathrm{nM} \mathrm{MB}$ was given (from $47 \pm 3 \mathrm{nmol} / \mathrm{min} /$ $\mathrm{mg}$ protein to $59 \pm 4 \mathrm{nmol} / \mathrm{min} / \mathrm{mg}$ protein), by $72 \%$ with $1 \mu \mathrm{M} \mathrm{MB}$ (from $47 \pm 3 \mathrm{nmol} / \mathrm{min} / \mathrm{mg}$ protein to $81 \pm 4 \mathrm{nmol} / \mathrm{min} / \mathrm{mg}$ protein) and by $104 \%$ with $2 \mu \mathrm{M} \mathrm{MB}$ (from $47 \pm 3 \mathrm{nmol} / \mathrm{min} / \mathrm{mg}$ protein to $96 \pm 4 \mathrm{nmol} / \mathrm{min} / \mathrm{mg}$ protein) (Fig. 2B). Addition of the ANT inhibitor CAT lowered the rate of ATP production nearly to the level of control (i.e. when only ADP and oligomycin were added) indicating that "excess" ATP is produced within the mitochondria and that ANT activity was necessary to detect SLP-mediated ATP production.

Glutamate-energised mitochondria exhibited moderate levels of basal SLP $(31 \pm 4)-(23 \pm 1)=8 \pm 2 \mathrm{nmol} / \mathrm{min} / \mathrm{mg}$ protein. $\mathrm{MB}(1 \mu \mathrm{M})$ elevated the rate of ATP synthesis from $31 \pm 4$ to $54 \pm 6 \mathrm{nmol} / \mathrm{min} / \mathrm{mg}$ protein (Fig. 2D). 
A

a) $\alpha-K G, A D P, C A T$

b) $\alpha-K G, A D P$, oligomycin, CAT

c) $\alpha-K G$, ADP, oligomycin, MB, CAT

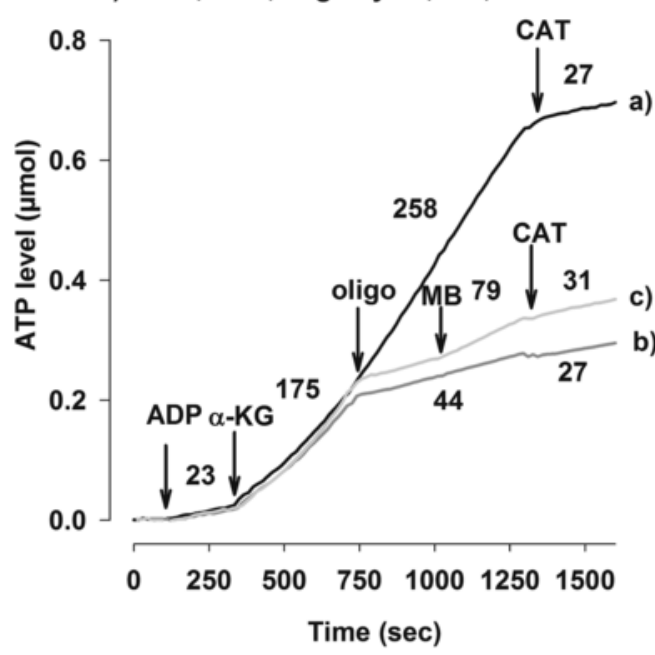

C

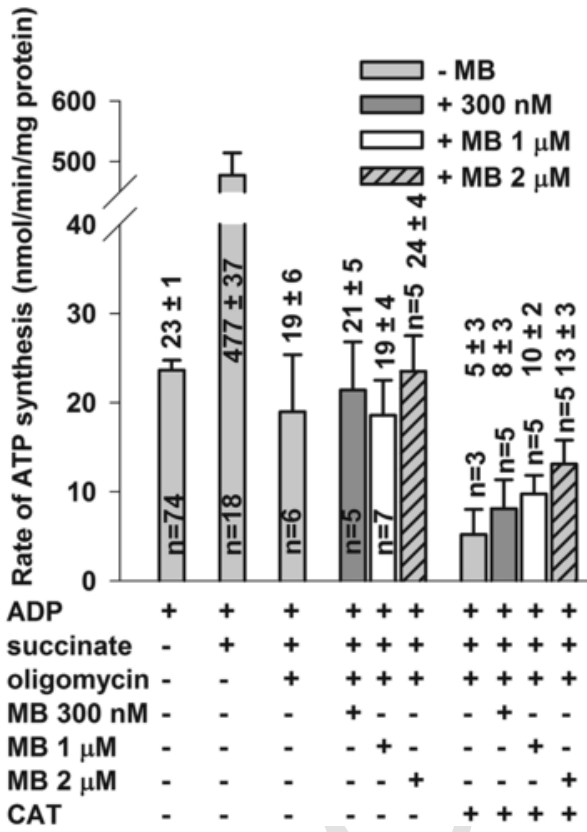

B

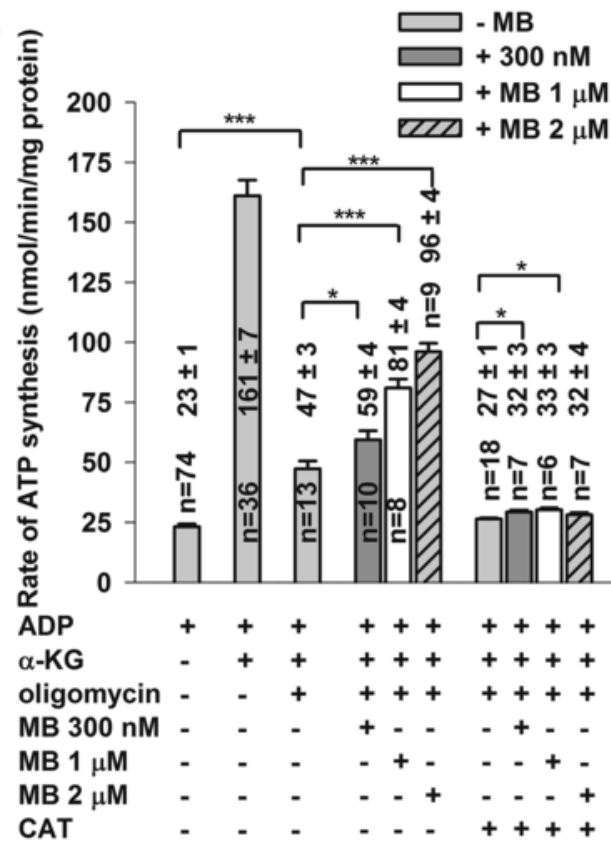

D

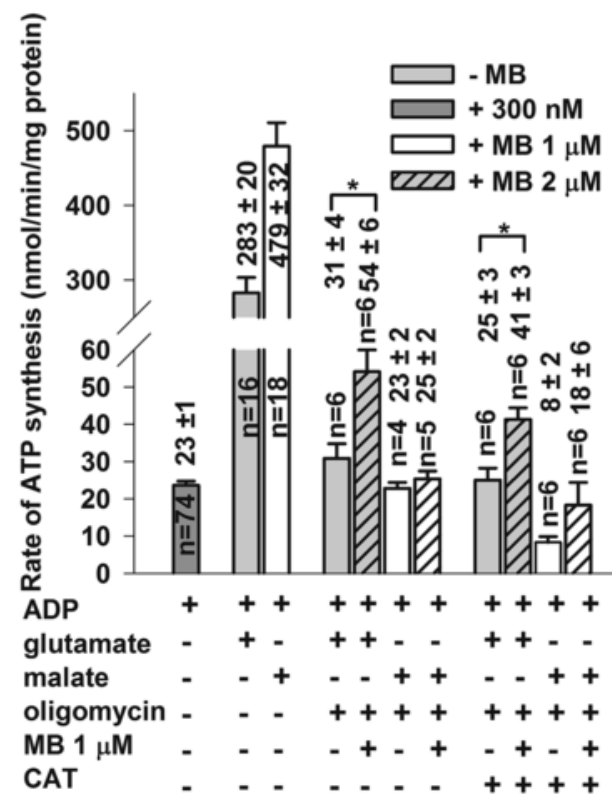

Fig. 2. The effect of $\mathrm{MB}$ on the rate of ATP synthesis in $\alpha$-ketoglutarate $(A, B)$, succinate $(C)$, in glutamate $(D)$ and malate $(D)$ supported brain mitochondria. AP5 $(200 \mu \mathrm{M})$ was added to each sample. Mitochondria $(0.05 \mathrm{mg} / \mathrm{ml})$ were incubated in the standard medium as described under Materials and methods. ADP $(2 \mathrm{mM})$, oligomycin $(2 \mu \mathrm{M})$, MB (300 nM, $1 \mu \mathrm{M}$, $2 \mu \mathrm{M})$ and carboxyatractilozide (CAT; $2 \mu \mathrm{M})$ were given as indicated. Original traces are shown in $(A)$ with numbers calculated as the rate of ATP synthesis in nmol/min $/ \mathrm{mg}$ protein in these particular experiments. The results are expressed as rate of ATP synthesis in nmol $/ \mathrm{min} / \mathrm{mg}$ protein mean $\pm \operatorname{SEM}(n>4)$ and written on the bars; ${ }^{*} p<0.05 ; * * * p<0.001$. The values $n$ in the figures refer to the number of repetitions in each group.

In succinate-energised mitochondria, no basal SLP was detected and the addition of MB did not enhance the rate of ATP generation in the presence of oligomycin. In the absence of MB the rate of ATP synthesis was found to be $19 \pm 6 \mathrm{nmol} / \mathrm{min} / \mathrm{mg}$ protein. In the presence of $300 \mathrm{nM} \mathrm{MB}, 21 \pm 5 \mathrm{nmol} / \mathrm{min} / \mathrm{mg}$ protein; with $1 \mu \mathrm{M} \mathrm{MB}$, $19 \pm 4 \mathrm{nmol} / \mathrm{min} / \mathrm{mg}$ protein and with $2 \mu \mathrm{M} \mathrm{MB}, 24 \pm 4 \mathrm{nmol} / \mathrm{min} / \mathrm{mg}$ protein rate of ATP production was detected after oligomycin was given (Fig. 2C). These results indicate that succinate does not support SLP in the TCA cycle.
Similarly to succinate, in malate-supported mitochondria a basal level of SLP was not detectable. Moreover, MB $(1 \mu \mathrm{M})$ did not increase the rate of ATP production in OXPHOS-inhibited malate-respiring mitochondria (Fig. 2D). These observations indicate that malate does not support SLP.

Uncouplers have been shown to stimulate mitochondrial SLP in ATP synthase-inhibited mitochondria (Rigoulet et al., 1985). As a control, the effect of the uncoupler FCCP was examined on ATP synthesis in $\alpha$-KG-supported, oligomycin-treated brain mitochondria. In 
the presence of oligomycin, the addition of the uncoupler $(50 \mathrm{nM}$ FCCP instead of MB) increased the rate of ATP synthesis from $47 \pm 3 \mathrm{nmol} / \mathrm{min} / \mathrm{mg}$ protein to $103 \pm 13 \mathrm{nmol} / \mathrm{min} / \mathrm{mg}$ protein in $\alpha-K G-$ respiring mitochondria (data not shown).

Supporting the data, the effects of MB and various mitochondrial inhibitors on the most important bioenergetic functions are summarized in (Table 1).

\subsection{Effects of $M B$ on the oxygen consumption in brain mitochondria supported by various substrates}

Oxygen consumption experiments followed a protocol which is identical, except for minor modifications, to that used for the ATP production measurements described under Section 3.2. Mitochondria were first energised with various respiratory substrates, then addition of ADP initiated state 3 respiration and OXPHOS, while oligomycin inhibited ATP synthase and MB stimulated electron transfer from $\mathrm{NADH}$ or $\mathrm{FADH}_{2}$ to cytochrome c. Experiments using $\alpha-\mathrm{KG}$ and succinate as respiratory substrates were terminated by addition of CAT, an inhibitor of ANT.

In oligomycin-treated, $\alpha-\mathrm{KG}$-energised mitochondria (Fig. 3A,B) MB stimulated the oxygen consumption in a dose-dependent manner. When mitochondria were challenged with MB, 57, 129, and $167 \%$ increases in oxygen consumption were observed using $300 \mathrm{nM} \mathrm{MB}$, $1 \mu \mathrm{M} \mathrm{MB}$, and $2 \mu \mathrm{M} \mathrm{MB}$, respectively, after ATP production had already been inhibited by oligomycin. The addition of CAT in the presence of MB triggered a moderate decrease in oxygen consumption.

In succinate-energised mitochondria, $300 \mathrm{nM} \mathrm{MB}, 1 \mu \mathrm{M} \mathrm{MB}$, and $2 \mu \mathrm{M}$ MB led to 93,108 , and $210 \%$ increase, of the oxygen consumption rate, respectively, in the presence of oligomycin (Fig. 3C). CAT induced no alteration in oxygen consumption which also indicated the absence of SLP.

In glutamate-energised mitochondria $1 \mu \mathrm{M}$ MB stimulated oxygen consumption by $100 \%$ from $24 \pm 4$ to $48 \pm 3 \mathrm{nmol} / \mathrm{min} / \mathrm{mg}$ protein provided that OXPHOS was inhibited by oligomycin (Fig. 3D). CAT lowered oxygen consumption from $48 \pm 3$ to $31 \pm 3 \mathrm{nmol} / \mathrm{min} / \mathrm{mg}$ protein in MB-treated mitochondria (data not shown).

As shown in Fig. 3D, $1 \mu \mathrm{M}$ MB increased the rate of $\mathrm{O}_{2}$ consumption from $40 \pm 2$ to $61 \pm 2 \mathrm{nmol} / \mathrm{min} / \mathrm{mg}$ protein (a $52 \%$ enhancement) in malate-respiring mitochondria. CAT induced no significant alteration in $\mathrm{O}_{2}$ consumption in the presence of $\mathrm{MB}$ (data not shown).

\subsection{Effects of $M B$ on the $N A D(P) H$ level}

The protocol for the $\mathrm{NAD}(\mathrm{P}) \mathrm{H}$ autofluorescence measurements followed the same steps as those of the 3.4. section. The respiratory substrate $(\alpha-K G)$ increased the mitochondrial NAD(P)H level (Fig.
4). The ADP-accelerated respiration was accompanied by a decreased steady-state level of NAD $(\mathrm{P}) \mathrm{H}$. In the presence of oligomycin, which augmented the mitochondrial $\mathrm{NAD}(\mathrm{P}) \mathrm{H}$ level, MB $(1 \mu \mathrm{M})$ significantly decreased the $\mathrm{NAD}(\mathrm{P}) \mathrm{H}$ autofluorescence in $\alpha-\mathrm{KG}$-respiring brain mitochondria (Fig. 4, trace c). In ATP synthase-inhibited mitochondria energised with $\alpha-K G$, CAT further attenuated the NAD $(\mathrm{P}) \mathrm{H}$ level in the presence of MB. In contrast, in the absence of MB the administration of CAT had no influence on the NAD $(\mathrm{P}) \mathrm{H}$ autofluorescence in respiration-impaired, $\alpha$-KG-energised mitochondria (Fig. 4, trace $b$ ). These experiments demonstrated the ability of MB to oxidize intramitochondrial NAD $(\mathrm{P}) \mathrm{H}$.

\subsection{Effects of $M B$ on the mitochondrial membrane potential $\left(\Delta \psi_{m}\right)$}

The $\Delta \psi_{\mathrm{m}}$ was measured semiquantitatively via safranin-O fluorescence in isolated mitochondria respiring on $\alpha-\mathrm{KG}$, succinate or glutamate (Fig. 5.). The experimental protocol of the $\Delta \psi_{\mathrm{m}}$ measurement was similar to that of the NAD $(\mathrm{P}) \mathrm{H}-\mathrm{level}$ measurement $(\mathrm{Sec}-$ tion 3.4). In the presence of respiratory substrates, the inner mitochondrial membrane became hyperpolarised. ADP was given, which decreased the $\Delta \psi_{\mathrm{m}}$, because the entry of protons through the $\mathrm{F}_{0} \mathrm{~F}_{1}$-ATPase lowered the proton motive force (Nicholls and Ferguson, 2013). Oligomycin increased the $\Delta \psi_{\mathrm{m}}$ due to the accumulation of protons in the intermembrane space. In $\alpha$-KG-supported mitochondria MB had no significant influence on the $\Delta \psi_{\mathrm{m}}$, whereas the subsequent addition of CAT slightly depolarised the $\Delta \psi_{\mathrm{m}}$ (Fig. $5 \mathrm{~A}$, trace $c$ ). Contrary to that, addition of CAT did not influence the $\Delta \psi_{\mathrm{m}}$ when MB was omitted (Fig. 5A, trace b). The MB-dependent unusual effect of CAT is explained in the Discussion. In succinate-energised mitochondria after the ATP-synthase was inhibited by oligomycin, the effect of MB was negligible (Fig. 5B, trace c). In contrast to that observed with $\alpha-K G$, the addition of CAT did not alter the $\Delta \psi_{\mathrm{m}}$ in the presence of MB for succinate-energised, OXPHOS-impaired mitochondria (Fig. 5B, trace b). Moreover, in glutamate-respiring mitochondria, MB added after oligomycin treatment also did not influence the $\Delta \psi_{\mathrm{m}}$ (Fig. 5C, trace c) but CAT led to depolarization in MB-treated mitochondria similarly to that found in $\alpha-\mathrm{KG}$-supported mitochondria (Fig. $5 \mathrm{C}$, trace $c$ ).

\subsection{Effects of $M B$ on mitochondrial bioenergetics in ATP-synthase- and complex I- inhibited mitochondria}

Complex I impairment is implicated in several neurodegenerative diseases (for review see (Bose and Beal, 2016; Chaturvedi and Beal, 2013)). The aim of the experiments was to reveal whether the enhancing effect of MB on SLP could also be detected in complex I-inhibited mitochondria. Rotenone was used to inhibit Complex I, in all

Table 1

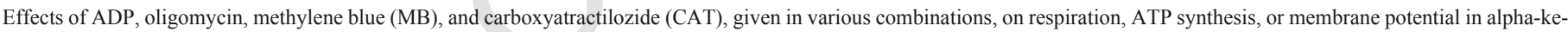

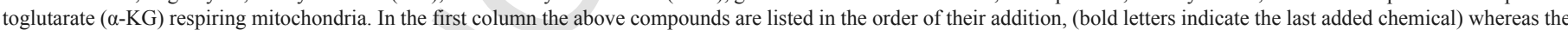
respective gross effects of that compund are indicated in the subsequent columns. Abbreviations: SLP, substrate-level phosphorylation.

\begin{tabular}{|c|c|c|c|}
\hline Compounds added to mitochondria & Respiration & ATP synthesis & Membrane potential \\
\hline$\alpha$-ketoglutarate & Basal respiration (state2) & No ATP synthesis & Hyperpolarisation \\
\hline$\alpha-K G+\mathbf{A D P}$ & Stimulated respiration (state3) & Maximal OXPHOS + SLP & Slight depolarisation \\
\hline $\begin{array}{l}\alpha-K G+\text { ADP } \\
+ \text { oligomycin }\end{array}$ & Inhibition & $\begin{array}{l}\text { Inhibitied OXPHOS, } \\
\text { decreased SLP }\end{array}$ & Hyperpolarisation \\
\hline$\alpha-\mathrm{KG}+\mathrm{ADP}+$ oligomycin $+\mathbf{M B}$ & Increase & $\begin{array}{l}\text { Inhibited OXPHOS, } \\
\text { stimulated SLP }\end{array}$ & No change \\
\hline$\alpha-\mathrm{KG}+\mathrm{ADP}+$ oligomycin $+\mathrm{MB}+\mathbf{C A T}$ & Decrease & $\begin{array}{l}\text { Inhibited OXPHOS } \\
\text { Inhibited SLP }\end{array}$ & Slight depolarisation \\
\hline$\alpha-\mathrm{KG}+\mathrm{ADP}+\mathbf{C A T}$ & Decrease & $\begin{array}{l}\text { Decreased OXPHOS } \\
\text { Decreased SLP }\end{array}$ & Hyperpolarisation \\
\hline
\end{tabular}


A

a) $\alpha-K G, A D P, C A T$

b) $\alpha-K G, A D P$, oligomycin, CAT

c) $\alpha-K G, A D P$, oligomycin, MB $1 \mu \mathrm{M}, \mathrm{CAT}$

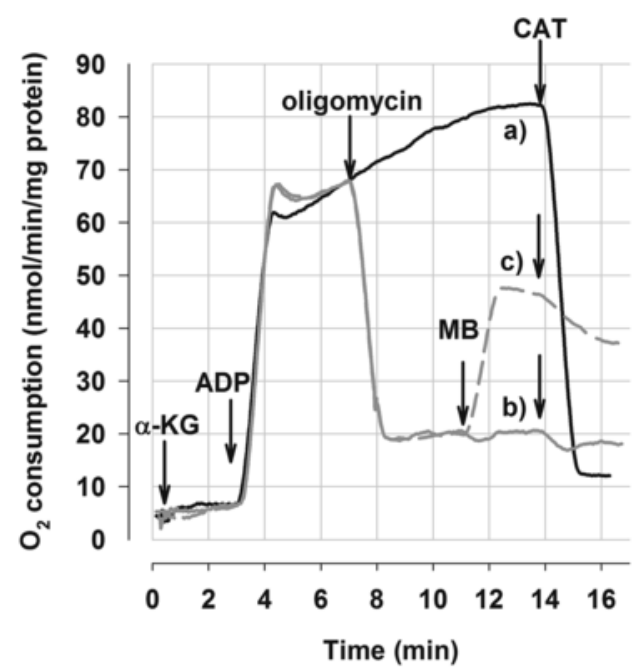

C $\square$ - MB

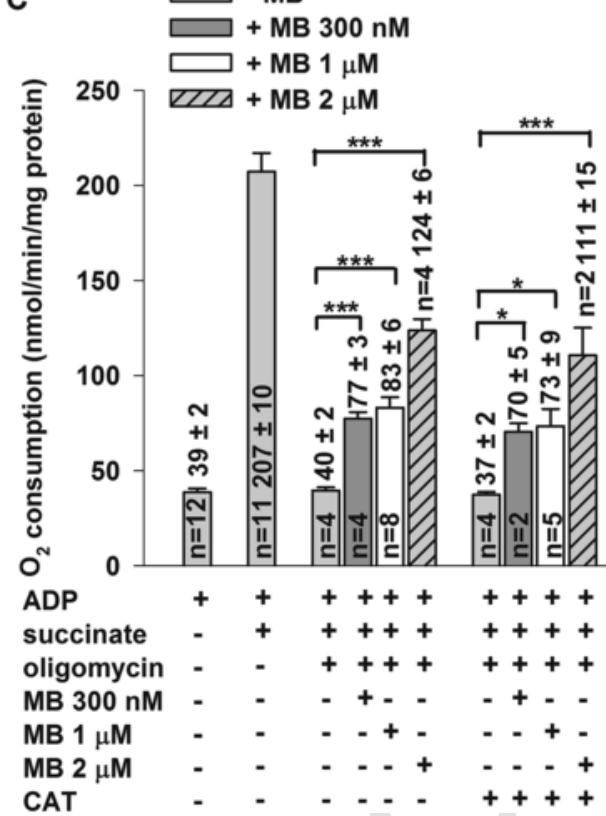

B

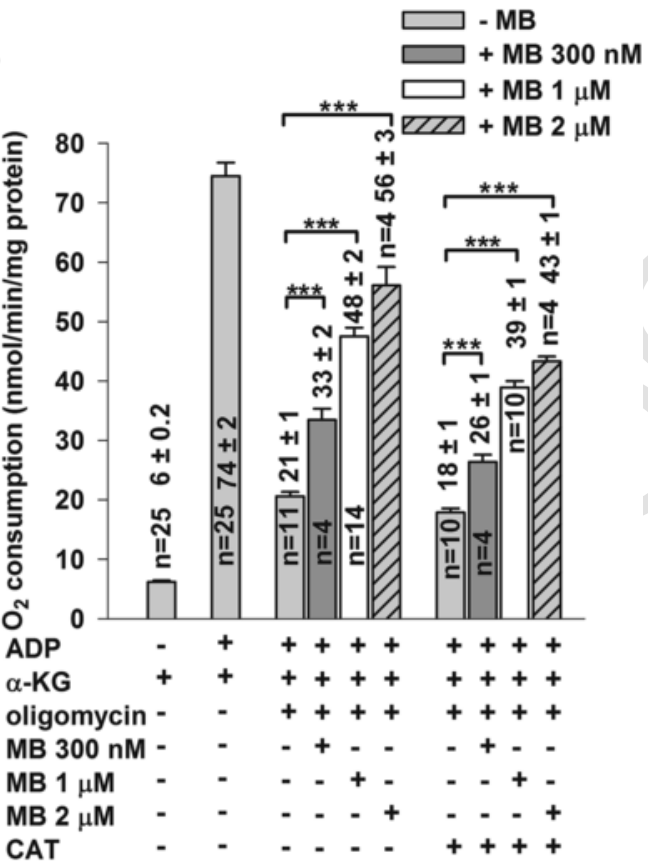

D

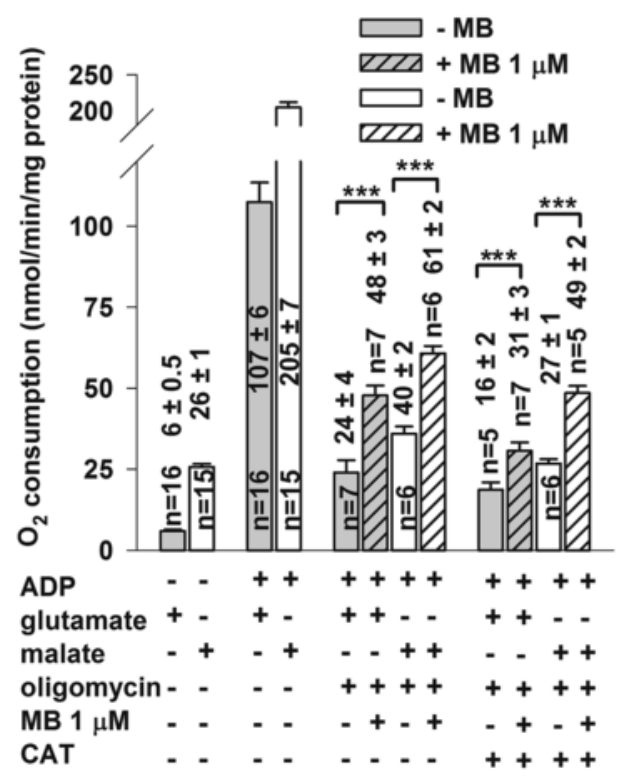

Fig. 3. The effect of $\mathrm{MB}$ on the rate of oxygen consumption in $\alpha$-ketoglutarate $(A ; B)$ and succinate $(C)$, glutamate $(D)$ and malate $(D)$ supported brain mitochondria. Representative experiments with original traces are shown in $(A)$. AP5 $(200 \mu \mathrm{M})$ was added to each sample. Mitochondria $(0.1 \mathrm{mg} / \mathrm{ml})$ were incubated in the standard medium as described under Materials and methods. ADP $(2 \mathrm{mM})$, oligomycin $(2 \mu \mathrm{M}), \mathrm{MB}(300 \mathrm{nM}, 1 \mu \mathrm{M}, 2 \mu \mathrm{M})$ and carboxyatractilozide (CAT; $2 \mu \mathrm{M})$ were given as indicated. The effect of MB $(1 \mu \mathrm{M})($ trace c) is compared with controls (without oligomycin and MB: trace $a$; and without MB: trace $b$ ). The results are expressed as the mean oxygen consumption in nmol/min/mg protein \pm SEM $(n>4)$ and written on the bars; ${ }^{*} p<0.05 ;{ }^{* * *} p<0.001$. The values $n$ in the figures refer to the number of repetitions in each group.

of the experiments. In the following experiments, oligomycin and the complex I inhibitor rotenone were applied in parallel.

\subsubsection{Effects of $M B$ on ATP synthesis}

Addition of ADP and $\alpha-K G$ or malate initiated OXPHOS. Oligomycin plus rotenone inhibited ATP-synthase and complex I which inhibitions were accompanied by the decrease of ATP production (Fig. 6A). Challenging the $\alpha-K G$-treated mitochondria with MB increased the rate of ATP synthesis by $59 \%$ (from $17 \pm 2$ to
$27 \pm 2 \mathrm{nmol} / \mathrm{min} / \mathrm{mg}$ protein) in case of $1 \mu \mathrm{M} \mathrm{MB}$ and by $282 \%$ (from $17 \pm 2$ to $65 \pm 10 \mathrm{nmol} / \mathrm{min} / \mathrm{mg}$ protein) with $2 \mu \mathrm{M} \mathrm{MB}$. However, in malate-energised mitochondria MB when added after oligomycin plus rotenone did not increase the rate of ATP generation (Fig. 6A).

\subsubsection{Effects of $M B$ on $\Delta \psi_{m}$}

In $\alpha-K G$ or malate-energised mitochondria, addition of the respiratory substrate hyperpolarised the mitochondrial membrane while 


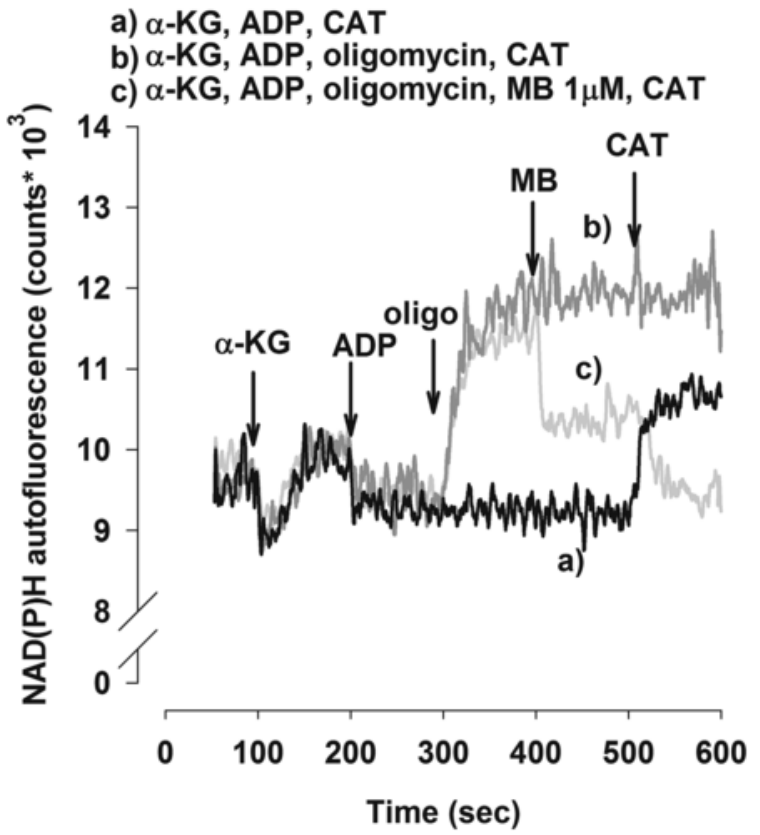

Fig. 4. The effect of MB on the steady-state level of NAD(P)H in $\alpha$-ketoglutarate-supported brain mitochondria. AP5 $(200 \mu \mathrm{M})$ was added to each sample. Mitochondria $(0.1 \mathrm{mg} / \mathrm{ml})$ were incubated in the standard medium as described under Materials and methods. ADP $(2 \mathrm{mM})$, oligomycin $(2 \mu \mathrm{M}), \mathrm{MB}(1 \mu \mathrm{M})$ and carboxyatractilozide $(\mathrm{CAT} ; 2 \mu \mathrm{M})$ were given as indicated. The effect of MB (trace c) is compared with controls (without oligomycin and MB: trace $a$; and without MB: trace $b$ )

ADP caused depolarisation. Oligomycin augmented the $\Delta \psi_{\mathrm{m}}$, as was seen previously (Fig. 5.), while rotenone decreased the $\Delta \psi_{\mathrm{m}}$ inhibiting the electron flow. $2 \mu \mathrm{M}$ MB restored the $\Delta \psi_{\mathrm{m}}$ both in $\alpha$-KG- (Fig. 6A) or malate-supported (Fig. 6B) mitochondria. At the end of each experiment FCCP was added to dissipate the $\Delta \psi_{\mathrm{m}}$.

\subsubsection{Effects of $M B$ on the steady-state level of $N A D(P) H$}

The protocol for the $\mathrm{NAD}(\mathrm{P}) \mathrm{H}$ autofluorescence measurements was identical to the one of the 3.6.2 section. Mitochondria were incubated in the presence of MB (Fig. 7A, trace $a$ ) or in the absence (Fig. 7A, trace $b$ ) of MB. As described in Section 3.4, in the absence of $\mathrm{MB}$ (Fig. 7A, trace $b$ ) $\alpha-\mathrm{KG}$ augmented the NAD(P)H fluorescence, while ADP lowered the steady-state level of $\mathrm{NAD}(\mathrm{P}) \mathrm{H}$ and oligomycin increased the NAD(P)H level. The subsequent addition of FCCP oxidized the NAD(P)H pool. Administration of the complex I inhibitor rotenone elevated the $\mathrm{NAD}(\mathrm{P}) \mathrm{H}$ level. On the contrary, the MB $(2 \mu \mathrm{M})$ which was present throughout the experiment (Fig. 7A, trace a) modified the $\mathrm{NAD}(\mathrm{P}) \mathrm{H}$ steady-state of mitochondria. In the presence of $\mathrm{MB}$ the changes in the $\mathrm{NAD}(\mathrm{P}) \mathrm{H}$ level were rather moderate relative to the control indicating the ability of $\mathrm{MB}$ to oxidize $\mathrm{NAD}(\mathrm{P}) \mathrm{H}$

\subsubsection{Mitochondrial accumulation of $M B$ in $\alpha-K G$-respiring mitochondria}

Owing to the aromatic rings and the positive charge of $\mathrm{MB}$, it is able to cross the mitochondrial membranes and accumulate in the mitochondrial matrix (Gabrielli et al., 2004). In order to verify the mitochondrial uptake of MB, the oxidized MB's absorbance was measured at $660 \mathrm{~nm}$ in the presence of isolated brain mitochondria (Fig. $7 \mathrm{~B})$. MB $(2 \mu \mathrm{M})$ was added to mitochondria at the beginning of each experiment. This was followed by the addition of $\alpha-K G$ which resulted in a drop in absorbance indicating the reduction (and uptake) of MB. OXPHOS was initiated by ADP resulting in a subtle elevation of level of oxidized MB. Administration of oligomycin caused membrane hyperpolarisation (Fig. 5A.), elevation of the NAD(P)H level (Fig. 4.) and an increase in the concentration of reduced MB. The uncoupler FCCP, increased the absorbance of MB to its maximum level, indicating the near complete oxidation of the dye. Challenging mitochondria with rotenone in the presence of FCCP resulted no change in the absorbance of MB. The measurements were always terminated by dithionite, which completely reduced MB.

\section{Discussion}

The aim of the present study was to examine the effects of MB on mitochondrial SLP. The substrate specificity of the MB-stimulated SLP was also investigated. Succinyl-CoA is a product of the $\alpha$-ketoglutarate dehydrogenase $(\alpha-\mathrm{KGDH})$-catalysed reaction in the TCA cycle. Succinyl-CoA ligase provides ATP via SLP for mitochondria even in the absence of oxygen (Weinberg et al., 2000) or when the respiratory chain is impaired (Kiss et al., 2013). Maintaining a low $\mathrm{NADH} / \mathrm{NAD}^{+}$ratio in the mitochondrial matrix is crucial for an effective SLP. A high NADH concentration inhibits the activity of the $\alpha-\mathrm{KGDH}$ (Smith et al., 1974) and hence the $\alpha-\mathrm{KGDH}$ under this condition cannot provide succinyl-CoA, a substrate for succinyl-CoA ligase. In anoxia or respiratory chain impairment, diaphorases are capable of sustaining an adequate level of $\mathrm{NAD}^{+}$for the $\alpha-\mathrm{KGDHc}$ via the oxidation of NADH (Chinopoulos et al., 2010; Kiss et al., 2013). Reduction in the $\alpha-\mathrm{KGDHc}$ activity, which is often associated with neurodegeneration (Gibson et al., 2000), which decreases the availability of succinyl-CoA for matrix SLP (Kiss et al., 2013). Data from the Chinopoulos group (Chinopoulos et al., 2010; Kiss et al., 2013) suggest that the SLP might be able to provide a rescue mechanism for cell survival via generating ATP independently from the respiratory chain in mitochondria; this mechanism can also prevent the reversal of the ANT and the mitochondrial consumption of cytosolic ATP.

To be able to selectively measure the SLP all possible ATP forming reactions had to be considered. In our experiments, the contribution of adenylate kinases (AKs) to mitochondrial ATP production was not negligible (Fig. 1.). To minimise this contribution, an inhibitor of AKs, AP5 $(200 \mu \mathrm{M})$ (Melnick et al., 1979) was used throughout the experiments. Without AP5, the ATP synthesis attributed to the AK isoenzymes decreased as a function of time (Fig. 1.), preventing the accurate detection of SLP. To eliminate the contribution of OXPHOS to ATP synthesis, the $\mathrm{F}_{0} \mathrm{~F}_{1}$-ATPase was inhibited by oligomycin throughout the experiments.

\subsection{The mitochondrial "target" of $M B$}

To be able to influence mitochondrial functions, $\mathrm{MB}$ has to enter the mitochondria. MB is able to cross the mitochondrial membranes in a $\Delta \psi_{\mathrm{m}}$-dependent manner and accumulate in the mitochondrial matrix (Gabrielli et al., 2004). In order to prove the mitochondrial translocation of $\mathrm{MB}$ in our systems, the absorbance of the oxidized $\mathrm{MB}$ was followed at $660 \mathrm{~nm}$ (Fig. 7B). In the presence of mitochondria, but in the absence of respiratory substrates $\mathrm{MB}$ remained fully oxidised (its absorbance was maximal). The addition of oligomycin was found to be coupled to the increased level of reduced MB, while this effect was eliminated by the addition of the uncoupler that reduced the uptake of MB via depolarisation of $\Delta \psi_{\mathrm{m}}$. A correlation was found between the oxidation-reduction states of $\mathrm{MB}$ and that of NAD(P)H (Fig. 7A,B); a higher NAD(P)H level appeared to be associated with a higher level of reduced $\mathrm{MB}$ (measured as the reduction of absorbance at $660 \mathrm{~nm}$ ): this correlation was eliminated by the addition of the uncoupler (the $\Delta \psi_{\mathrm{m}}$ collapsed, which prevented the ac- 
A

a) $\alpha$-KG, ADP, CAT, FCCP

b) $\alpha-K G$, ADP, oligo, CAT, FCCP

c) $\alpha-K G, A D P$, oligo, MB $1 \mu \mathrm{M}, \mathrm{CAT}, \mathrm{FCCP}$

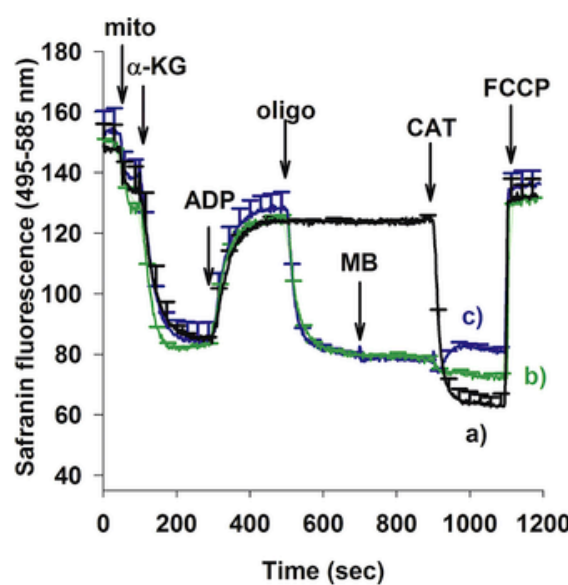

C a) glutamate, $A D P, C A T$

b) glutamate, $A D P$, oligo, $C A T$

c) glutamate, ADP, oligo, MB $1 \mu \mathrm{M}, \mathrm{CAT}$ mito

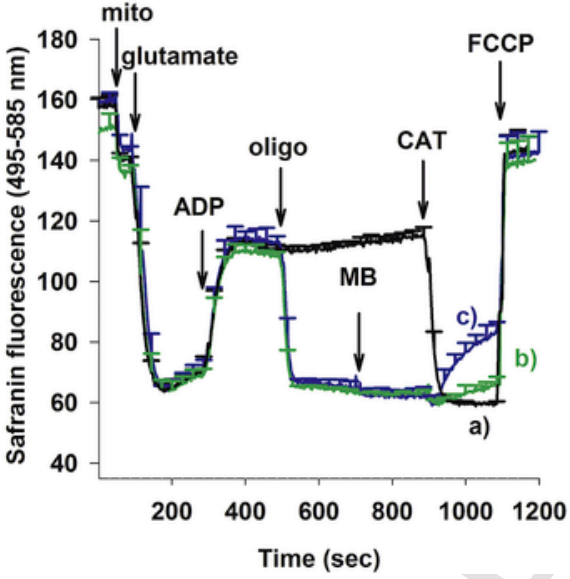

B

a) succinate, ADP, CAT, FCCP

b) succinate, ADP, oligo, CAT, FCCP

c) succinate, ADP, oligo, MB $1 \mu \mathrm{M}, \mathrm{CAT}, \mathrm{FCCP}$

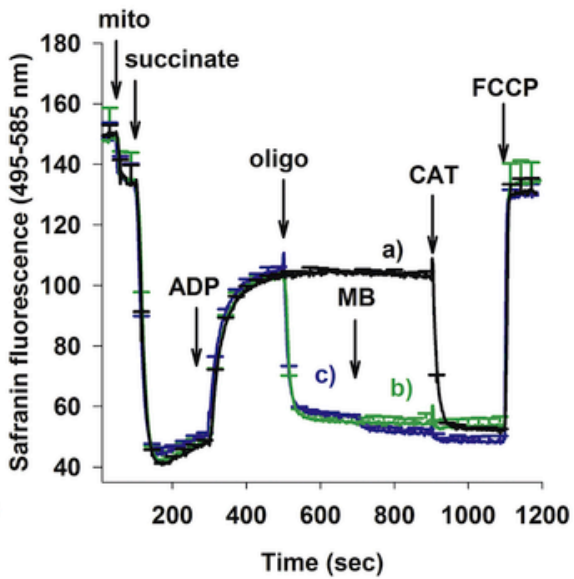

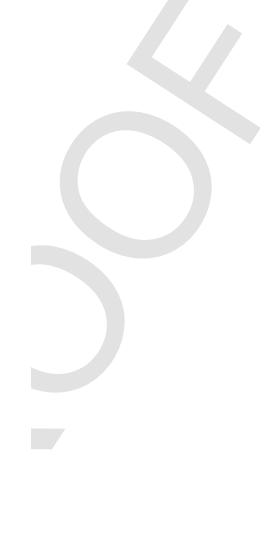

Fig. 5. The effect of MB on safranin fluorescence indicating $\Delta \psi_{\mathrm{m}}$ in $\alpha$-ketoglutarate $(A)$, succinate $(B)$, and glutamate $(C)$ supported brain mitochondria. AP5 (200 $\left.\mu \mathrm{M}\right)$ was added to each sample. Mitochondria $(0.1 \mathrm{mg} / \mathrm{ml})$ were incubated in the standard medium as described under Materials and methods. ADP $(2 \mathrm{mM})$, oligomycin $(2 \mu \mathrm{M})$, MB $(1 \mu \mathrm{M})$, carboxyatractilozide (CAT; $2 \mu \mathrm{M})$ and FCCP $(250 \mathrm{nM})$ were given as indicated. The effect of MB (trace c) is compared with controls (without oligomycin and MB: trace a; without MB: trace $b$ ). Each trace represents an average \pm SEM of three independent experiments.

cumulation of $\mathrm{MB})$. Therefore, in the presence of the uncoupler (FCCP) rotenone increased the $\mathrm{NAD}(\mathrm{P}) \mathrm{H}$ level but no reduction of MB could be detected (Fig. 7A,B). These observations can be explained by the fact that low concentrations of $\mathrm{MB}(0.5-2 \mu \mathrm{M})$ were capable of accepting electrons from $\mathrm{NAD}(\mathrm{P}) \mathrm{H}$ and $\mathrm{FADH}_{2}$ providing an alternative pathway for transferring electrons onto cytochrome $\mathrm{c}$ when the respiratory chain was impaired (Atamna et al., 2008; Wen et al., 2011). This alternative electron transfer mechanism bypasses complexes I and/or III, therefore changes the efficiency of proton pumping: in case of $\mathrm{NADH}$ from $10 \mathrm{H}^{+} / \mathrm{NADH}$ to $2 \mathrm{H}^{+} / \mathrm{NADH}$, and with succinate- $\mathrm{FADH}_{2}$ from $6 \mathrm{H}^{+} / \mathrm{FADH}_{2}$ to $2 \mathrm{H}^{+} / \mathrm{FADH}_{2}$ (Tretter et al., 2014). The decrease in pumping efficiency likely stimulates oxidation with all of the respiratory substrates undergoing oxido-reduction.

In this study, various respiratory substrates were applied to energise isolated guinea pig brain mitochondria. The effects of $\mathrm{MB}$ on mitochondrial SLP, oxygen consumption, $\Delta \psi_{\mathrm{m}}$ and $\mathrm{NAD}(\mathrm{P}) \mathrm{H}$ autofluorescence were investigated in $\alpha-\mathrm{KG}$-, succinate-, glutamate- or malate-supported mitochondria. To support understanding a short summary of the effects of the chemicals used in this study was provided in Table 1.

\subsection{The effects of $M B$ on mitochondria utilising $\alpha-K G$}

In $\alpha$-KG-supported mitochondria in the presence of oligomycin, the rate of ATP synthesis $(47 \pm 3 \mathrm{nmol} / \mathrm{min} / \mathrm{mg}$ protein) was higher than that observed in the absence of $\alpha-K G(23 \pm 1 \mathrm{nmol} / \mathrm{min} / \mathrm{mg}$ protein) and in the presence of $2 \mathrm{mM}$ ADP (Fig. 2B). This elevation was attributed to the basal $\alpha-K G$-stimulated mitochondrial SLP. MB added at various concentrations caused a dose-dependent increase of SLP. MB-induced SLP was most efficient with $\alpha-\mathrm{KG}$ as compared to other respiratory substrates. In $\alpha-\mathrm{KG}$-supported and oligomycin-treated mitochondria challenge with $\mathrm{MB}$ resulted in the decrease of $\mathrm{NAD}(\mathrm{P}) \mathrm{H}$ autofluorescence. Subsequent addition of CAT further reduced the $\mathrm{NAD}(\mathrm{P}) \mathrm{H}$ level in the MB-treated mitochondria (Fig. 4, 


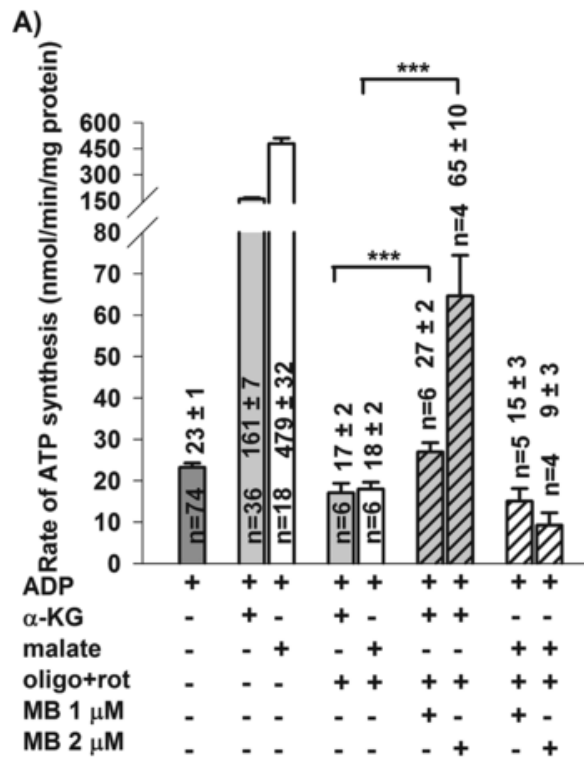

B)

a) $\alpha-K G$, ADP, oligo, rotenone, FCCP

b) $\alpha$-KG, ADP, oligo, rotenone, MB $2 \mu \mathrm{M}, \mathrm{FCCP}$

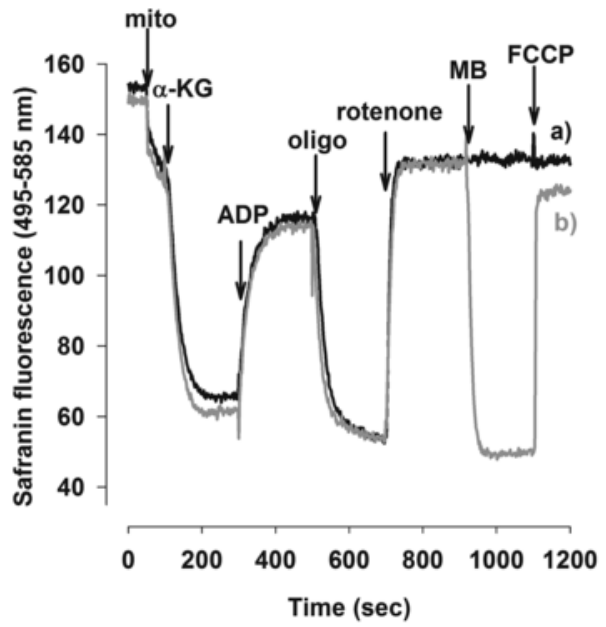

C)

a) malate, ADP, oligo, rotenone, FCCP

b) malate, ADP, oligo, rotenone, MB $2 \mu \mathrm{M}$, FCCP

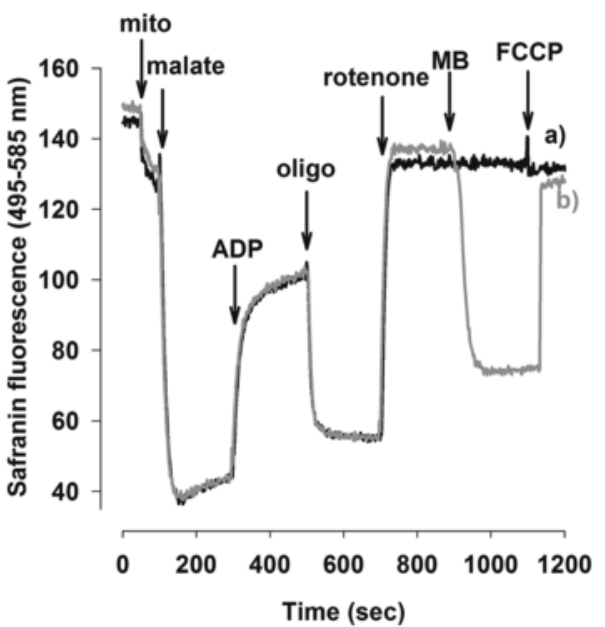

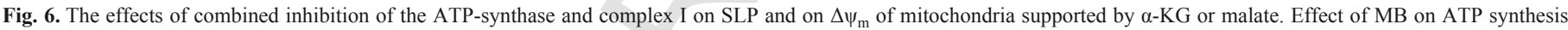

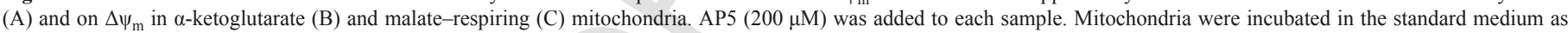

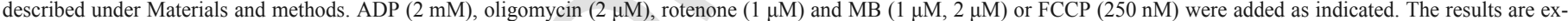

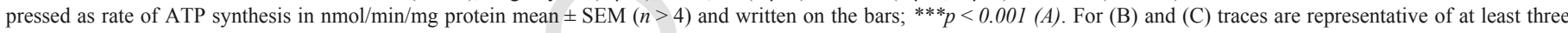
independent experiments. The values $n$ in the figures refer to the number of repetitions in each group.

trace c). This phenomenon indicated that the inhibition of ANT kept SLP-produced ATP inside the mitochondria; accumulation of ATP could inhibit the $\alpha$-KGDHc (Lawlis and Roche, 1981) and result in decreased $\mathrm{NADH}+\mathrm{H}^{+}$levels. However, in the absence of MB the $\mathrm{NAD}(\mathrm{P}) \mathrm{H}$ level did not diminish after giving CAT (Fig. 4, trace b). This demonstrated that basal SLP likely did not produce enough ATP to inhibit the $\alpha$-KGDHc. This explanation was further supported by our observations which demonstrated that in the absence of MB the inhibition of the ANT did not significantly decrease respiration. However, in ATP-synthase-inhibited mitochondria respiring on $\alpha$-KG MB stimulated respiration and the inhibition of the ANT decreased the rate of oxygen consumption (Fig. 3B). This phenomenon demonstrates the inhibition of the $\alpha$-KGDHc by SLP-mediated ATP.
Additionally, these observations appeared to be also supported by the $\Delta \psi_{\mathrm{m}}$ measurements. In the presence of MB CAT caused depolarisation, which could be explained by the above mentioned inhibitory effect of ATP on the $\alpha-\mathrm{KGDHc}$ that results in a decrease in $\mathrm{NADH}+\mathrm{H}^{+}$production (Fig. 5A, trace c). In the absence of MB, mitochondria responded to ANT inhibition by slight hyperpolarisation because the normal forward function of the ANT leads to a decreased proton gradient (Fig. 5A, trace b) (Brand et al., 2005; Chinopoulos et al., 2009; Klingenberg, 2008; Villiers et al., 1979).

\subsection{The effects of $M B$ on mitochondria utilising succinate}

Mitochondria supported by succinate reacted differently to MB treatment. The lack of stimulation of basal SLP in succinate-treated mitochondria (Fig. 2C), which is different from that observed with 
A)

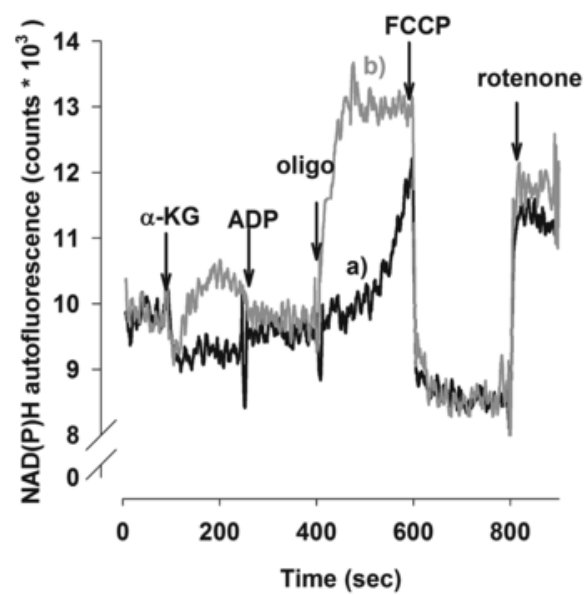

B)

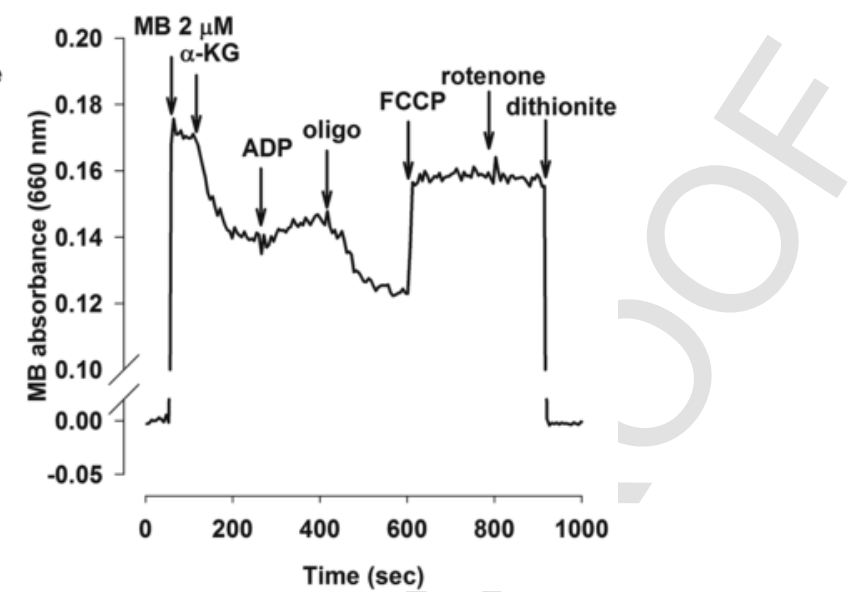

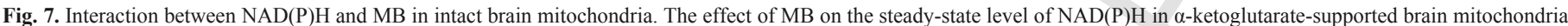

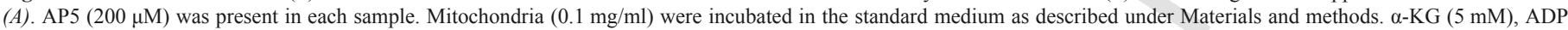

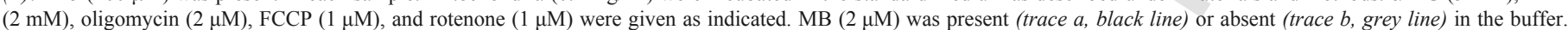

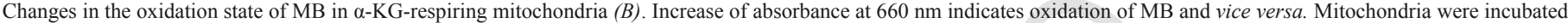
under the conditions of Fig. 7A, trace a. At $900 \mathrm{sec}$ dithionite was added as indicated. For both $(A)$ and $(B)$ traces are representatives of at least three independent experiments.

$\alpha-K G$, supported the results of a previous study (Kiss et al., 2013) wherein succinate did not contribute to the mitochondrial SLP. Although $\mathrm{MB}$ was able to increase the rate of oxygen consumption, this stimulation was not associated with an increased ATP production. Furthermore, CAT did not inhibit the MB-stimulated respiration indicating that succinate oxidation was not associated with ATP production under these conditions. In succinate-supported mitochondria MB did not decrease significantly the NAD(P)H level, and CAT did not decrease the steady-state level of NAD(P)H (data not shown). In mitochondria treated with oligomycin and MB CAT did not depolarize $\Delta \psi_{\mathrm{m}}$ (Fig. 5B). These findings suggest that in the presence of succinate and MB the SLP-mediated ATP production is not active.

\subsection{The effects of $M B$ on mitochondria utilising glutamate}

In glutamate-energised mitochondria, basal level of SLP was detected $(31 \pm 4)-(23 \pm 1)=8 \pm 2 \mathrm{nmol} / \mathrm{min} / \mathrm{mg}$ protein, while MB could further stimulated SLP in the presence of oligomycin (Fig. 2D). The smaller rate of SLP observed in glutamate-supported mitochondria relative to $\alpha$-KG-respiring mitochondria, is explained by the two-step dehydrogenation of glutamate to succinyl-CoA via the successive actions of glutamate dehydrogenase and the $\alpha$-KGDHc: oxidation of 1 mol NADH allows formation of $1 \mathrm{~mol}$ of succinyl-CoA in $\alpha$-KG-supported mitochondria. However, the oxidation of $1 \mathrm{~mol}$ of NADH results in only $0.5 \mathrm{~mol}$ of succinyl-CoA in the oxidative catabolism of glutamate. The decreased yield of succinyl-CoA formation was reflected in the reduced rate of the SLP in glutamate-supported mitochondria (Fig. 2D). Measurements for $\Delta \psi_{\mathrm{m}}$ and oxygen consumption carried out in the presence of glutamate showed similarities to those performed in the presence of $\alpha-K G$. We concluded that regarding the SLP there is only a quantitative difference exists between glutamate and $\alpha-\mathrm{KG}$-energised mitochondria.

\subsection{The effects of $M B$ on mitochondria utilising malate}

Mitochondria energised by malate did not support SLP in either the absence or presence of MB. Considering the role of malate in the TCA cycle the probability of malate contributing to the formation of
succinyl-CoA and to SLP is low (Kiss et al., 2013). Similarly to other respiratory substrates investigated in this study, in malate-oxidising mitochondria, $\mathrm{MB}(1 \mu \mathrm{M})$ was able to stimulate oxygen consumption in the presence of oligomycin (Fig. 3D). However, this stimulation of respiration did not result in an enhanced ATP production (Fig. 2D).

\subsection{Relationship between oxygen consumption, $M B$, and ATP production in oligomycin-treated mitochondria}

As described above, the contributions of the different respiratory substrates to the SLP-mediated ATP production are dissimilar. Considering the unequal oxidation rates of the respiratory substrates, the efficiency of the SLP-mediated ATP production in relation to oxygen consumption should be further examined. We calculated a parameter whose bioenergetic interpretation is similar to the one of $\mathrm{P} / \mathrm{O}$ ratio; the $\mathrm{P} / \mathrm{O}$ ratio is frequently used in mitochondrial physiology, and quantifies the efficacy of ATP production in relation to oxygen consumption (Chance and Williams, 1956; Lee et al., 1996; Lehninger and Smith, 1949). Taking into account that the $\mathrm{P} / \mathrm{O}$ ratio (ATP production $/ \mathrm{O}_{2}$ consumption) calculations use nanogram atoms of oxygen instead of nanomoles (Lee et al., 1996), the $\Delta \mathrm{P} / \Delta \mathrm{O}$ ratio after treatment with $2 \mu \mathrm{M}$ MB was $(96-47) /(2 *(56-21)=0.7$ for $\alpha-K G$ and $(24-19) /$ $2 *(124-40)=0.03$ for succinate. This was calculated from the difference of the rate of ATP generation detected after MB treatment and the basal SLP and divided by the difference of the rate of $\mathrm{O}_{2}$ consumption detected after $\mathrm{MB}$ administration and oligomycin addition: the maximum $\mathrm{P} / \mathrm{O}$ ratio in the absence of OXPHOS is one. The difference between the theoretical maximum 1 and the calculated ratio 0.7 can be explained by the less than $100 \%$ efficacy of ATP detection, the possibility that mitochondria may use a certain portion of the produced ATP themselves, and the increased formation of reactive oxygen species in MB-treated mitochondria (Tretter et al., 2014).

To illustrate the effects of MB on oxygen consumption and on ATP production, data taken from Figs. 2 and 3 were compared in Fig. 8: the dependence of ATP production on oxygen consumption was plotted. Our findings demonstrate that from the respiratory substrates investigated in this study only $\alpha-K G$ and glutamate reacted with an increased rate of ATP synthesis to MB-stimulated oxidation. Contrary 


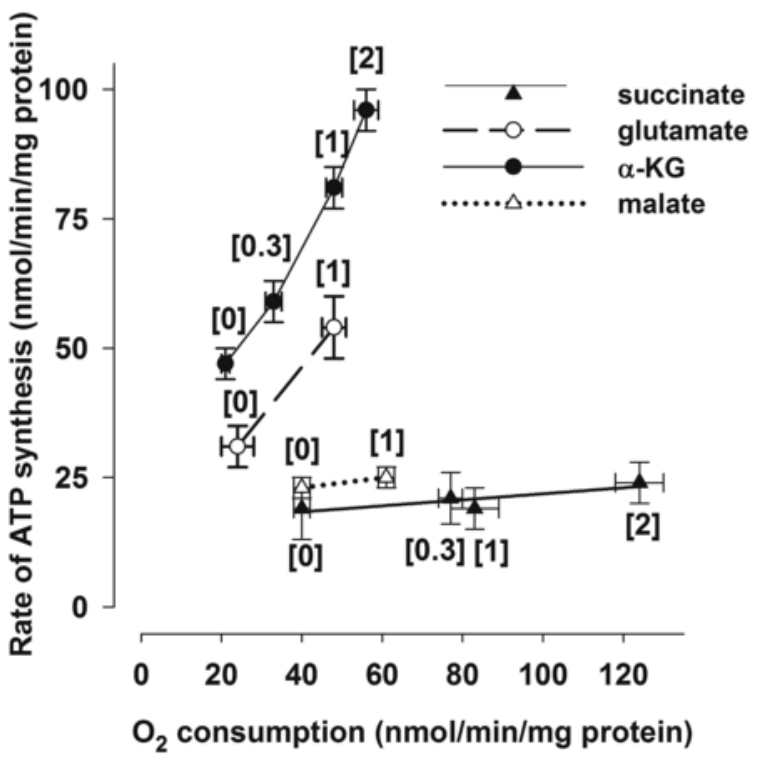

Fig. 8. Relationship between the rate of ATP production and oxygen consumption in the presence or absence of MB in oligomycin-treated mitochondria supported by various respiratory substrates: $\alpha$-ketoglutarate (black circles, solid line), succinate (black triangles, solid line), glutamate (white circles, long dash) and malate (white triangles, dotted) energised mitochondria. Mitochondria were incubated in the standard medium as described under Materials and methods. ADP $(2 \mathrm{mM})$, oligomycin $(2 \mu \mathrm{M})$ and $\mathrm{MB}$ $(0-2 \mu \mathrm{M})$ were given. $\mathrm{MB}$ concentrations $(\mu \mathrm{M})$ are indicated in brackets. Data were taken from Fig. 2 and 3., respectively. The results are expressed as the mean oxygen consumption and as the mean ATP production in $\mathrm{nmol} / \mathrm{min} / \mathrm{mg}$ protein $\pm \operatorname{SEM}(n>4)$.

to that MB similarly stimulated oxygen consumption but hardly increased ATP production in succinate and malate-energised mitochondria (Fig. 8).

\subsection{The beneficial effects of $M B$ in combined inhibition of respiratory complex I and $F_{1} F_{o}$ ATP synthase}

MB seems to be a neuroprotective agent in neurodegenerative disease models (Atamna et al., 2012; Poteet et al., 2012). In neurodegenerative diseases mitochondrial functions are usually impaired (Chaturvedi and Beal, 2013). The most important mitochondrial functions associated with bioenergetics are the synthesis of ATP and contribution to the maintenance of ionic homeostasis of the cells. Inhibition of respiratory complex I prevents the building up of $\Delta \psi_{\mathrm{m}}$ and the OXPHOS (Nicholls and Ferguson, 2013). These two mitochondrial functions mutually dependent upon each other. Without $\Delta \psi_{\mathrm{m}}$ there is no OXPHOS and the reversal of ATP synthase function, ATP hydrolysis and proton pumping can be associated with $\Delta \psi_{\mathrm{m}}$ formation (Chinopoulos and Adam-Vizi, 2010). In mitochondria with the respiratory complex I and ATP synthase inhibited MB partially restored ATP synthesis and $\Delta \psi_{\mathrm{m}}$.

Herein we demonstrate that MB increases the SLP-coupled ATP production in complex I-inhibited isolated brain mitochondria. This phenomenon can contribute to the neuroprotective effects of MB in neurodegenerative diseases. In oligomycin plus rotenone-treated $\alpha$-KG-respiring mitochondria MB greatly stimulated ATP production (Fig. 6.). However, in malate-energised mitochondria MB did not increase the rate of ATP synthesis, which refers to the substrate-dependency of the mitochondrial SLP. The effects of different mitochondrial substrates on SLP in the presence of MB are summarized (Fig. 9). Contrary to the substrate-specific stimulation of the SLP, the oligomycin plus rotenone induced collapse of $\Delta \psi_{\mathrm{m}}$ was repolarized

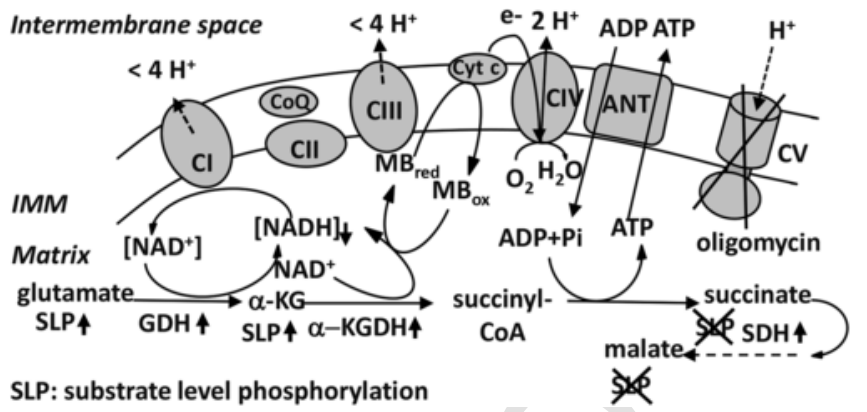

Fig. 9. Summary of MB-stimulated changes in oxidative phosphorylation inhibited brain mitochondria. Mitochondria supported by Complex I (CI) (glutamate or alpha-ketoglutarate or malate) or Complex II (CII) succinate substrate, respectively. MB accepts electrons from NADH and donates them to cytochrome c, thus the efficacy of proton pumping is decreased. The decreased level of mitochondrial NADH (or FADH with succinate substrate) stimulates the activity of dehydrogenases and increases the flux of the citrate cycle. Those substrates that can form succinyl-CoA $(\alpha-\mathrm{KG}$ and glutamate) will contribute to SLP, while succinate and malate are localized "downstream" from the succinyl-CoA hence do not contribute to succinyl-CoA formation for SLP. Abbreviations: $\mathrm{CI}, \mathrm{CII}, \mathrm{CIII}, \mathrm{CIV}$ : respiratory chain complexes, $\mathrm{CV}$ : mitochondrial $\mathrm{F}_{0} \mathrm{~F}_{1}$ ATP-ase, GDH: glutamate dehydrogenase, $\alpha-\mathrm{KGDHc}$ : alpha-ketoglutarate dehydrogenase complex, SDH: succinate dehydrogenase.

by MB even with that substrate (malate) which did not support SLP (Fig. 6C). Taking into account that the directionality of the ATP and ADP transport by the ANT is both concentration- and $\Delta \psi_{\mathrm{m}}$-dependent (for a mathematical model see (Chinopoulos, 2011a; Metelkin et al., 2009)) the repolarising effect of MB might prevent the hydrolysis of cytosolic ATP by stimulating the oxidation of those substrates which do not support SLP.

\section{Conclusion}

The ability of MB to accept and transfer electrons within the mitochondrial electron transport system combined with the positive charge and lipophilic character of the compound make the $\Delta \psi_{\mathrm{m}}$-dependent mitochondrial enrichment of MB possible. MB stimulates the oxidation of $\mathrm{NADH}$, hence it can also increase/restore the metabolic flux inside the citric acid cycle, which is a prerequisite of SLP. Here we conclude that the enhancement of SLP and the restitution of $\Delta \psi_{\mathrm{m}}$ may have beneficial effects under pathological conditions and could contribute to the possible neuroprotective effects of MB.

\section{Funding}

This work was supported by the Hungarian Brain Research Program (KTIA_13_NAP-A-III/6), OTKA (NK 81983), and the Hungarian Academy of Sciences (MTA TKI 02001), all to Vera Adam-Vizi.

\section{Acknowledgements}

Authors are indebted to Dr Attila Ambrus for carefully reading the manuscript and making several helpful comments. The authors thank Katalin Takács and Andrea Várnagy for the excellent technical assistance.

\section{Appendix A. Supplementary data}

Supplementary data related to this article can be found at http://dx. doi.org/10.1016/j.neuropharm.2017.05.009. 


\section{References}

Akerman, K.E., Wikstrom, M.K., 1976. Safranine as a probe of the mitochondrial membrane potential. FEBS Lett. 68, 191-197.

Atamna, H., Mackey, J., Dhahbi, J.M., 2012. Mitochondrial pharmacology: electron transport chain bypass as strategies to treat mitochondrial dysfunction. Biofactors $38,158-166$.

Atamna, H., Nguyen, A., Schultz, C., Boyle, K., Newberry, J., Kato, H., Ames, B.N., 2008. Methylene blue delays cellular senescence and enhances key mitochondrial biochemical pathways. FASEB J. 22, 703-712.

Barkulis, S.S., Lehninger, A.L., 1951. Myokinase and the adenine nucleotide specificity in oxidative phosphorylations. J. Biol. Chem. 190, 339-344.

Bose, A., Beal, M.F., 2016. Mitochondrial dysfunction in Parkinson's disease. J. Neurochem. 139 (Suppl. 1), 216-231.

Bradford, M.M., 1976. A rapid and sensitive method for the quantitation of microgram quantities of protein utilizing the principle of protein-dye binding. Anal. Biochem. 72, 248-254.

Brand, M.D., Pakay, J.L., Ocloo, A., Kokoszka, J., Wallace, D.C., Brookes, P.S., Cornwall, E.J., 2005. The basal proton conductance of mitochondria depends on adenine nucleotide translocase content. Biochem. J. 392, 353-362.

Bruns, G.A., Regina, V.M., 1977. Adenylate kinase 2, a mitochondrial enzyme. Biochem. Genet. 15, 477-486.

Chance, B., Williams, G.R., 1956. The respiratory chain and oxidative phosphorylation. Adv. Enzymol. Relat. Subj. Biochem. 17, 65-134.

Chaturvedi, R.K., Beal, F.M., 2013. Mitochondrial diseases of the brain. Free Radic. Biol. Med. 63, 1-29.

Chinopoulos, C., 2011a. The "B space" of mitochondrial phosphorylation. J. Neurosci. Res. 89, 1897-1904.

Chinopoulos, C., 2011b. Mitochondrial consumption of cytosolic ATP: not so fast FEBS Lett. 585, 1255-1259.

Chinopoulos, C., Adam-Vizi, V., 2010. Mitochondria as ATP consumers in cellular pathology. Biochim. Biophys. Acta 1802, 221-227.

Chinopoulos, C., Gerencser, A.A., Mandi, M., Mathe, K., Torocsik, B., Doczi, J., Turiak, L., Kiss, G., Konrad, C., Vajda, S., Vereczki, V., Oh, R.J., Adam-Vizi, V., 2010. Forward operation of adenine nucleotide translocase during F0F1-ATPase reversal: critical role of matrix substrate-level phosphorylation. FASEB J. 24, 2405-2416.

Chinopoulos, C., Vajda, S., Csanady, L., Mandi, M., Mathe, K., Adam-Vizi, V., 2009. A novel kinetic assay of mitochondrial ATP-ADP exchange rate mediated by the ANT. Biophys. J. 96, 2490-2504.

Di, Y., He, Y.L., Zhao, T., Huang, X., Wu, K.W., Liu, S.H., Zhao, Y.Q., Fan, M., Wu, L.Y., Zhu, L.L., 2015. Methylene blue reduces acute cerebral ischemic injury via the induction of mitophagy. Mol. Med. 21, 420-429.

Dixon, M., 1971. The acceptor specificity of flavins and flavoproteins. II. Free flavins. Biochim. Biophys. Acta 226, 259-268.

Draize, J.H., 1933. Sodium tetrathionate and methylene blue in cyanide and carbon monoxide poisoning. Science 78, 145.

Eckert, G.P., Renner, K., Eckert, S.H., Eckmann, J., Hagl, S., Abdel-Kader, R.M., Kurz, C., Leuner, K., Muller, W.E., 2012. Mitochondrial dysfunction-a pharmacological target in Alzheimer's disease. Mol. Neurobiol. 46, 136-150.

Figueira, T.R., Melo, D.R., Vercesi, A.E., Castilho, R.F., 2012. Safranine as a fluorescent probe for the evaluation of mitochondrial membrane potential in isolated organelles and permeabilized cells. Methods Mol. Biol. 810, 103-117.

Gabrielli, D., Belisle, E., Severino, D., Kowaltowski, A.J., Baptista, M.S., 2004. Binding, aggregation and photochemical properties of methylene blue in mitochondrial suspensions. Photochem Photobiol. 79, 227-232.

Gibson, G.E., Park, L.C., Sheu, K.F., Blass, J.P., Calingasan, N.Y., 2000. The alpha-ketoglutarate dehydrogenase complex in neurodegeneration. Neurochem. Int. 36, 97-112.

Guttman, P., Ehrlich, P., 1891. Ueber die Wirkung des Methylenblau bei Malaria. Berl. Klin. Wochenschr. 39, 953-956.

Heldt, H.W., Schwalbach, K., 1967. The participation of GTP-AMP-P transferase in substrate level phosphate transfer of rat liver mitochondria. Eur. J. Biochem. 1, 199-206.

Johnson, J.D., Mehus, J.G., Tews, K., Milavetz, B.I., Lambeth, D.O., 1998. Genetic evidence for the expression of ATP- and GTP-specific succinyl-CoA synthetases in multicellular eucaryotes. J. Biol. Chem. 273, 27580-27586.

Kiss, G., Konrad, C., Doczi, J., Starkov, A.A., Kawamata, H., Manfredi, G., Zhang, S.F., Gibson, G.E., Beal, M.F., Adam-Vizi, V., Chinopoulos, C., 2013. The negative impact of alpha-ketoglutarate dehydrogenase complex deficiency on matrix substrate-level phosphorylation. FASEB J. 27, 2392-2406.

Klingenberg, M., 2008. The ADP and ATP transport in mitochondria and its carrier. Biochim. Biophys. Acta 1778, 1978-2021.

Lambeth, D.O., Tews, K.N., Adkins, S., Frohlich, D., Milavetz, B.I., 2004. Expression of two succinyl-CoA synthetases with different nucleotide specificities in mammalian tissues. J. Biol. Chem. 279, 36621-36624.
Lawlis, V.B., Roche, T.E., 1981. Inhibition of bovine kidney alpha-ketoglutarate dehydrogenase complex by reduced nicotinamide adenine dinucleotide in the presence or absence of calcium ion and effect of adenosine 5'-diphosphate on reduced nicotinamide adenine dinucleotide inhibition. Biochemistry 20, 2519-2524.

Lee, C.P., Gu, Q., Xiong, Y., Mitchell, R.A., Ernster, L., 1996. P/O ratios reassessed mitochondrial $\mathrm{P} / \mathrm{O}$ ratios consistently exceed 1.5 with succinate and 2.5 with NAD-linked substrates. FASEB J. 10, 345-350.

Lehninger, A.L., Smith, S.W., 1949. Efficiency of phosphorylation coupled to electron transport between dihydrodiphosphopyridine nucleotide and oxygen. J. Biol. Chem. 181, 415-429.

Mansouri, A., Lurie, A.A., 1993. Concise review: methemoglobinemia. Am. J. Hematol. $42,7-12$.

McCord, J.M., Fridovich, I., 1970. The utility of superoxide dismutase in studying free radical reactions. II. The mechanism of the mediation of cytochrome $\mathrm{c}$ reduction by a variety of electron carriers. J. Biol. Chem. 245, 1374-1377.

Melnick, R.L., Rubenstein, C.P., Motzkin, S.M., 1979. Measurement of mitochondrial oxidative phosphorylation: selective inhibition of adenylate kinase activity by P1,P5-di-(adenosine-5')-pentaphosphate. Anal. Biochem. 96, 7-11.

Metelkin, E., Demin, O., Kovacs, Z., Chinopoulos, C., 2009. Modeling of ATP-ADP steady-state exchange rate mediated by the adenine nucleotide translocase in isolated mitochondria. FEBS J. 276, 6942-6955.

Nicholls, D.G., Ferguson, S.J., 2013. Bioenergetics 4, fourth ed. Academic Press.

Nobumoto, M., Yamada, M., Song, S., Inouye, S., Nakazawa, A., 1998. Mechanism of mitochondrial import of adenylate kinase isozymes. J. Biochem. 123, 128-135.

Ostergaard, E., 2008. Disorders caused by deficiency of succinate-CoA ligase. J. Inherit. Metab. Dis. 31, 226-229.

Paban, V., Manrique, C., Filali, M., Maunoir-Regimbal, S., Fauvelle, F., Alescio-Lautier, B., 2014. Therapeutic and preventive effects of methylene blue on Alzheimer's disease pathology in a transgenic mouse model. Neuropharmacology 76 (Pt A), 68-79.

Panayiotou, C., Solaroli, N., Karlsson, A., 2014. The many isoforms of human adenylate kinases. Int. J. Biochem. Cell Biol. 49, 75-83.

Pesta, D., Gnaiger, E., 2012. High-resolution respirometry: OXPHOS protocols for human cells and permeabilized fibers from small biopsies of human muscle. Methods Mol. Biol. 810, 25-58.

Peter, C., Hongwan, D., Kupfer, A., Lauterburg, B.H., 2000. Pharmacokinetics and organ distribution of intravenous and oral methylene blue. Eur. J. Clin. Pharmacol. 56, 247-250.

Poteet, E., Winters, A., Yan, L.J., Shufelt, K., Green, K.N., Simpkins, J.W., Wen, Y., Yang, S.H., 2012. Neuroprotective actions of methylene blue and its derivatives. PLoS One 7, e48279.

Rafael, J., Wiemer, G., Hohorst, H.J., 1974. Mitochondria from brown adipose tissue: influence of albumin, guanine nucleotides and of substrate level phosphorylation on the internal adenine nucleotide pattern. Hoppe Seylers Z Physiol. Chem. 355, 341-352.

Rigoulet, M., Velours, J., Guerin, B., 1985. Substrate-level phosphorylation in isolated yeast mitochondria. Eur. J. Biochem. 153, 601-607.

Rosenthal, R.E., Hamud, F., Fiskum, G., Varghese, P.J., Sharpe, S., 1987. Cerebral ischemia and reperfusion: prevention of brain mitochondrial injury by lidoflazine. J. Cereb. Blood Flow. Metab. 7, 752-758.

Schirmer, R.H., Adler, H., Pickhardt, M., Mandelkow, E., 2011. Lest we forget you-methylene blue. Neurobiol. Aging 32. 2325 e2327-2316.

Smith, C.M., Bryla, J., Williamson, J.R., 1974. Regulation of mitochondrial alpha-ketoglutarate metabolism by product inhibition at alpha-ketoglutarate dehydrogenase. J. Biol. Chem. 249, 1497-1505.

Smith, R.E., Roberts, J.C., Hittelman, K.J., 1966. Nonphosphorylating respiration of mitochondria from brown adipose tissue of rats. Science 154, 653-654.

Sontag, E.M., Lotz, G.P., Agrawal, N., Tran, A., Aron, R., Yang, G., Necula, M., Lau, A., Finkbeiner, S., Glabe, C., Marsh, J.L., Muchowski, P.J., Thompson, L.M., 2012. Methylene blue modulates huntingtin aggregation intermediates and is protective in Huntington's disease models. J. Neurosci. 32, 11109-11119.

Tretter, L., Adam-Vizi, V., 2007. Moderate dependence of ROS formation on DeltaP sim in isolated brain mitochondria supported by NADH-linked substrates. Neurochem. Res. 32, 569-575.

Tretter, L., Horvath, G., Holgyesi, A., Essek, F., Adam-Vizi, V., 2014. Enhanced hydrogen peroxide generation accompanies the beneficial bioenergetic effects of methylene blue in isolated brain mitochondria. Free Radic. Biol. Med. 77, 317-330

Villiers, C., Michejda, J.W., Block, M., Lauquin, G.J., Vignais, P.V., 1979. The electrogenic nature of ADP/ATP transport in inside-out submitochondrial particles. Biochim. Biophys. Acta 546, 157-170.

Weinberg, J.M., Venkatachalam, M.A., Roeser, N.F., Nissim, I., 2000. Mitochondrial dysfunction during hypoxia/reoxygenation and its correction by anaerobic metabolism of citric acid cycle intermediates. Proc. Natl. Acad. Sci. U. S. A. 97, 2826-2831

Wen, Y., Li, W., Poteet, E.C., Xie, L., Tan, C., Yan, L.J., Ju, X., Liu, R., Qian, H., Marvin, M.A., Goldberg, M.S., She, H., Mao, Z., Simpkins, J.W., Yang, S.H., 2011 
Alternative mitochondrial electron transfer as a novel strategy for neuroprotection.

J. Biol. Chem. 286, 16504-16515.

Williamson, J.R., Corkey, B.E., 1979. Assay of citric acid cycle intermediates and related compounds-update with tissue metabolite levels and intracellular distribution. Methods Enzymol. 55, 200-222.

Zhang, X., Rojas, J.C., Gonzalez-Lima, F., 2006. Methylene blue prevents neurodegeneration caused by rotenone in the retina. Neurotox. Res. 9, 47-57. 